Second-order necessary conditions in Pontryagin form for optimal control problems

J. Frédéric Bonnans, Xavier Dupuis, Laurent Pfeiffer

\title{
RESEARCH
}

REPORT

$\mathrm{N}^{\circ} 8306$

May 2013

Project-Team COMMANDS 



\title{
Give
}

\section{Second-order necessary conditions in Pontryagin form for optimal control problems}

\author{
J. Frédéric Bonnans* Xavier Dupuis*, Laurent Pfeiffer* \\ Project-Team COMMANDS
}

Research Report n 8306 - May 2013 - 37pages

\begin{abstract}
In this report, we state and prove first- and second-order necessary conditions in Pontryagin form for optimal control problems with pure state and mixed control-state constraints. We say that a Lagrange multiplier of an optimal control problem is a Pontryagin multiplier if it is such that Pontryagin's minimum principle holds, and we call optimality conditions in Pontryagin form those which only involve Pontryagin multipliers. Our conditions rely on a technique of partial relaxation, and apply to Pontryagin local minima.
\end{abstract}

Key-words: Optimal control; pure state and mixed control-state constraints; Pontryagin's principle; Pontryagin multipliers; second-order necessary conditions; partial relaxation.

The research leading to these results has received funding from the EU 7th Framework Programme (FP7PEOPLE-2010-ITN), under GA number 264735-SADCO, and from the Gaspard Monge Program for Optimization and operations research (PGMO).

* Inria-Saclay and CMAP, Ecole Polytechnique, Route de Saclay, 91128 Palaiseau Cedex, France. frederic.bonnans@inria.fr, xavier.dupuis@cmap.polytechnique.fr, laurent.pfeiffer@polytechnique.edu.

Emails:

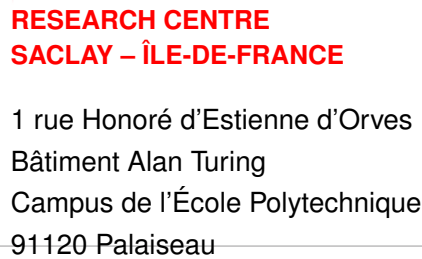




\section{Conditions nécessaires du second ordre sous forme Pontryaguine pour des problèmes de commande optimale}

Résumé : Dans ce rapport, nous énonçons et prouvons des conditions nécessaires du premier et second ordre sous forme Pontryaguine pour des problèmes de commande optimale avec contraintes pures sur l'état et mixtes sur l'état et la commande. Nous appelons multiplicateur de Pontryaguine tout multiplicateur de Lagrange pour lequel le principe de Pontryaguine est satisfait et parlons de conditions d'optimalité sous forme Pontryaguine si elles ne font intervenir que des multiplicateurs de Pontryaguine. Nos conditions s'appuient sur une technique de relaxation partielle et sont valables pour des minima de Pontryaguine.

Mots-clés : Commande optimale; contraintes pures sur l'état et contraintes mixtes sur l'état et la commande; principe de Pontryaguine; multiplicateurs de Pontryaguine; conditions nécessaires du second ordre; relaxation partielle. 


\section{Introduction}

The optimization theory in Banach spaces, in particular optimality conditions of order one [27, 30 and two [11, 21, 23, applies to optimal control problems. With this approach, constraints of various kind can be considered, and optimality conditions are derived for weak local minima of optimal control problems. Second-order necessary and sufficient conditions are thereby obtained by Stefani and Zezza [29] in the case of mixed control-state equality constraints, or by Bonnans and Hermant [6] in the case of pure state and mixed control-state constraints. These optimality conditions always involve Lagrange multipliers.

Another class of optimality conditions, necessary and of order one, for optimal control problems comes from Pontryagin's minimum principle. Formulated in the historical book [26] for basic problems, including first-order pure state constraints, this principle has then been extended by many authors. Mixed control-state constraints enter for example the framework developed by Hestenes [19, whereas pure state, and later pure state and mixed control-state, constraints are treated in early Russian references such as the works of Milyutin and Dubovitskii [15, 16], as highlighted by Dmitruk [12. Let us mention the survey by Hartl et al. [18] and its bibliography for more references on Pontryagin's principles.

Second-order optimality conditions are said in this article to be in Pontryagin form if they only involve Lagrange multipliers for which Pontryagin's minimum principle holds. This restriction to a subset of multipliers is a challenge for necessary conditions, and enables sufficient conditions to give strong local minima. To our knowledge, such conditions have been stated for the first time, under the name of quadratic conditions, for problems with mixed control-state equality constraints by Milyutin and Osmolovskii [24. Proofs are given by Osmolovskii and Maurer [25], under a restrictive full-rank condition for the mixed equality constraints, that could not for instance be satisfied by pure state constraints.

The main novelty of this paper is to provide second-order necessary conditions in Pontryagin form for optimal control problems with pure state and mixed control-state constraints. We use the same technique as Dmitruk in his derivation of Pontryagin's principle for a general optimal control problem [12]: a partial relaxation of the problem, based on the sliding modes introduced by Gamkrelidze [17]. These convexifications of the set of admissible velocities furnish a sequence of auxiliary optimal control problems, and at the limit, necessary conditions appear to be in Pontryagin form. We thereby get our own version of Pontryagin's minimum principle, as firstorder necessary conditions. Then, combining the partial relaxation with a reduction approach [5, 20 and a density argument [3], we obtain second-order necessary conditions in Pontryagin form for a Pontryagin local minimum of our problem. This technique requires to consider a variant of the previous auxiliary problems, but not to compute any envelope-like effect of Kawasaki [21. Another result that is worth being mentioned is the second-order necessary conditions for a local solution of an abstract optimization problem, that we apply to the partially relaxed problems. We derive them directly on a large set of directions in $L^{2}$, which then simplifies the density argument, compared with [3], and avoid a flaw that we will mention in the proof of the density result in [6].

Second-order sufficient conditions for strong local minima of similar optimal control problems constitute another work by the same authors [4. They rely on an extension of the decomposition principle of Bonnans and Osmolovskii [7, and on the reduction approach. Quadratic growth for a strong local minimum is then characterized.

The paper is organized as follows. In Section 2 we set our optimal control problem and define various notions of multipliers and of minima. Section 3 is devoted to the first-order necessary conditions: they are stated, under the form of Pontryagin's minimum principle, in Section 3.1. our partial relaxation approach is detailed in Section 3.2 and then used to prove the first-order 
conditions in Section 3.3. Section 4 is devoted to the second-order necessary conditions: they are stated in Section 4.1, and proved in Section 4.2 by partial relaxation combined with reduction and density. We have postponed our abstract optimization results to Appendix A.1, the proof of an approximation result needed for the partial relaxation to Appendix A.2, a qualification condition to Appendix A.3 and an example about Pontryagin's principle to Appendix A.4.

Notations For a function $h$ that depends only on time $t$, we denote by $h_{t}$ its value at time $t$, by $h_{i, t}$ the value of its $i$ th component if $h$ is vector-valued, and by $h$ its derivative. For a function $h$ that depends on $(t, x)$, we denote by $D_{t} h$ and $D_{x} h$ its partial derivatives. We use the symbol $D$ without any subscript for the differentiation w.r.t. all variables except $t$, e.g. $D h=D_{(u, y)} h$ for a function $h$ that depends on $(t, u, y)$. We use the same convention for higher order derivatives.

We identify the dual space of $\mathbb{R}^{n}$ with the space $\mathbb{R}^{n *}$ of $n$-dimensional horizontal vectors. Generally, we denote by $X^{*}$ the dual space of a topological vector space $X$. Given a convex subset $K$ of $X$ and a point $x$ of $K$, we denote by $T_{K}(x)$ and $N_{K}(x)$ the tangent and normal cone to $K$ at $x$, respectively; see [8, Section 2.2.4] for their definition.

We denote by $|\cdot|$ both the Euclidean norm on finite-dimensional vector spaces and the cardinal of finite sets, and by $\|\cdot\|_{s}$ and $\|\cdot\|_{q, s}$ the standard norms on the Lesbesgue spaces $L^{s}$ and the Sobolev spaces $W^{q, s}$, respectively.

We denote by $B V([0, T])$ the space of functions of bounded variation on the closed interval $[0, T]$. Any $h \in B V([0, T])$ has a derivative $\mathrm{d} h$ which is a finite Radon measure on $[0, T]$ and $h_{0}\left(\right.$ resp. $\left.h_{T}\right)$ is defined by $h_{0}:=h_{0_{+}}-\mathrm{d} h(0)$ (resp. $h_{T}:=h_{T_{-}}+\mathrm{d} h(T)$ ). Thus $B V([0, T])$ is endowed with the following norm: $\|h\|_{B V}:=\|\mathrm{d} h\|_{\mathcal{M}}+\left|h_{T}\right|$. See [2, Section 3.2] for a rigorous presentation of $B V$.

All vector-valued inequalities have to be understood coordinate-wise.

\section{Setting}

\subsection{The optimal control problem}

Consider the state equation

$$
\dot{y}_{t}=f\left(t, u_{t}, y_{t}\right) \quad \text { for a.a. } t \in(0, T) .
$$

Here, $u$ is a control which belongs to $\mathcal{U}, y$ is a state which belongs to $\mathcal{Y}$, where

$$
\mathcal{U}:=L^{\infty}\left(0, T ; \mathbb{R}^{m}\right), \quad \mathcal{Y}:=W^{1, \infty}\left(0, T ; \mathbb{R}^{n}\right),
$$

and $f:[0, T] \times \mathbb{R}^{m} \times \mathbb{R}^{n} \rightarrow \mathbb{R}^{n}$ is the dynamics. Consider constraints of various types on the system: the mixed control-state constraints, or mixed constraints

$$
c\left(t, u_{t}, y_{t}\right) \leq 0 \text { for a.a. } t \in(0, T),
$$

the pure state constraints, or state constraints

$$
g\left(t, y_{t}\right) \leq 0 \quad \text { for a.a. } t \in(0, T),
$$

and the initial-final state constraints

$$
\left\{\begin{array}{l}
\Phi^{E}\left(y_{0}, y_{T}\right)=0 \\
\Phi^{I}\left(y_{0}, y_{T}\right) \leq 0 .
\end{array}\right.
$$


Here $c:[0, T] \times \mathbb{R}^{m} \times \mathbb{R}^{n} \rightarrow \mathbb{R}^{n_{c}}, g:[0, T] \times \mathbb{R}^{n} \rightarrow \mathbb{R}^{n_{g}}, \Phi^{E}: \mathbb{R}^{n} \times \mathbb{R}^{n} \rightarrow \mathbb{R}^{n_{\Phi} E}, \Phi^{I}: \mathbb{R}^{n} \times \mathbb{R}^{n} \rightarrow$ $\mathbb{R}^{n_{\Phi} I}$. Consider finally the cost function $\phi: \mathbb{R}^{n} \times \mathbb{R}^{n} \rightarrow \mathbb{R}$. The optimal control problem is then

$$
\min _{(u, y) \in \mathcal{U} \times \mathcal{Y}} \phi\left(y_{0}, y_{T}\right) \quad \text { subject to } 2.1-2.5 \text {. }
$$

\subsection{Definitions and assumptions}

Similarly to [29, Definition 2.1], we introduce the following Carathéodory-type regularity notion:

Definition 2.1. We say that $\varphi:[0, T] \times \mathbb{R}^{m} \times \mathbb{R}^{n} \rightarrow \mathbb{R}^{s}$ is uniformly quasi- $C^{k}$ iff

(i) for a.a. $t,(u, y) \mapsto \varphi(t, u, y)$ is of class $C^{k}$, and the modulus of continuity of $(u, y) \mapsto$ $D^{k} \varphi(t, u, y)$ on any compact of $\mathbb{R}^{m} \times \mathbb{R}^{n}$ is uniform w.r.t. $t$.

(ii) for $j=0, \ldots, k$, for all $(u, y), t \mapsto D^{j} \varphi(t, u, y)$ is essentially bounded.

Remark 2.2. If $\varphi$ is uniformly quasi- $C^{k}$, then $D^{j} \varphi$ for $j=0, \ldots, k$ are essentially bounded on any compact, and $(u, y) \mapsto D^{j} \varphi(t, u, y)$ for $j=0, \ldots, k-1$ are locally Lipschitz, uniformly w.r.t. $t$. In particular, if $f$ is uniformly quasi- $C^{1}$, then by Cauchy-Lipschitz theorem, for any $\left(u, y^{0}\right) \in \mathcal{U} \times \mathbb{R}^{n}$, there exists a unique $y \in \mathcal{Y}$ such that 2.1 holds and $y_{0}=y^{0}$; we denote it by $y\left[u, y^{0}\right]$.

The minimal regularity assumption through all the paper is the following:

Assumption 1. The mappings $f, c$ and $g$ are uniformly quasi- $C^{1}, g$ is continuous, $\Phi^{E}, \Phi^{I}$ and $\phi$ are $C^{1}$.

We call a trajectory any pair $(u, y) \in \mathcal{U} \times \mathcal{Y}$ such that $(2.1)$ holds. We say that a trajectory is feasible for problem $(P)$ if it satisfies constraints $(2.3)-(2.5)$, and denote by $F(P)$ the set of feasible trajectories. We define the Hamiltonian and the augmented Hamiltonian respectively by

$$
H[p](t, u, y):=p f(t, u, y), \quad H^{a}[p, \nu](t, u, y):=p f(t, u, y)+\nu c(t, u, y),
$$

for $(p, \nu, t, u, y) \in \mathbb{R}^{n *} \times \mathbb{R}^{n_{c} *} \times[0, T] \times \mathbb{R}^{m} \times \mathbb{R}^{n}$. We define the end points Lagrangian by

$$
\Phi[\beta, \Psi]\left(y_{0}, y_{T}\right):=\beta \phi\left(y_{0}, y_{T}\right)+\Psi \Phi\left(y_{0}, y_{T}\right),
$$

for $\left(\beta, \Psi, y_{0}, y_{T}\right) \in \mathbb{R} \times \mathbb{R}^{n_{\Phi} *} \times \mathbb{R}^{n} \times \mathbb{R}^{n}$, where $n_{\Phi}=n_{\Phi^{E}}+n_{\Phi^{I}}$ and $\Phi=\left(\begin{array}{c}\Phi^{E} \\ \Phi^{I}\end{array}\right)$.

We denote

$$
K_{c}:=L^{\infty}\left(0, T ; \mathbb{R}_{-}^{n_{c}}\right), \quad K_{g}:=C\left([0, T] ; \mathbb{R}_{-}^{n_{g}}\right), \quad K_{\Phi}:=\{0\}_{\mathbb{R}^{n}{ }^{E}} \times \mathbb{R}_{-}^{n_{\Phi} I},
$$

so that the constraints 2.3$)-(2.5)$ can be rewritten as

$$
c(\cdot, u, y) \in K_{c}, \quad g(\cdot, y) \in K_{g}, \quad \Phi\left(y_{0}, y_{T}\right) \in K_{\Phi} .
$$

Recall that the dual space of $C\left([0, T] ; \mathbb{R}^{n_{g}}\right)$ is the space $\mathcal{M}\left([0, T] ; \mathbb{R}^{n_{g} *}\right)$ of finite vector-valued Radon measures. We denote by $\mathcal{M}\left([0, T] ; \mathbb{R}^{n_{g}{ }^{*}}\right)_{+}$the cone of positive measures in this dual space. Let

$$
E:=\mathbb{R} \times \mathbb{R}^{n_{\Phi} *} \times L^{\infty}\left(0, T ; \mathbb{R}^{n_{c^{*}}}\right) \times \mathcal{M}\left([0, T] ; \mathbb{R}^{n_{g^{*}}}\right)
$$

and let $\|\cdot\|_{E}$ be defined, for any $\lambda=(\beta, \Psi, \nu, \mu) \in E$, by

$$
\|\lambda\|_{E}:=|\beta|+|\Psi|+\|\nu\|_{1}+\|\mu\|_{\mathcal{M}} .
$$

$\mathrm{RR} \mathrm{n}^{\circ} 8306$ 
Let $(\bar{u}, \bar{y}) \in F(P)$. Let $N_{K_{c}}$ be the set of elements in the normal cone to $K_{c}$ at $c(\cdot, \bar{u}, \bar{y})$ that belong to $L^{\infty}\left(0, T ; \mathbb{R}^{n_{c} *}\right)$, i.e.

$$
N_{K_{c}}(c(\cdot, \bar{u}, \bar{y})):=\left\{\nu \in L^{\infty}\left(0, T ; \mathbb{R}_{+}^{n_{c^{*}}}\right): \nu_{t} c\left(t, \bar{u}_{t}, \bar{y}_{t}\right)=0 \text { for a.a. } t\right\} .
$$

Let $N_{K_{g}}$ be the normal cone to $K_{g}$ at $g(\cdot, \bar{y})$, i.e.

$$
N_{K_{g}}(g(\cdot, \bar{y})):=\left\{\mu \in \mathcal{M}\left([0, T] ; \mathbb{R}^{n_{g} *}\right)_{+}: \int_{[0, T]}\left(\mathrm{d} \mu_{t} g\left(t, \bar{y}_{t}\right)\right)=0\right\} .
$$

Let $N_{K_{\Phi}}$ be the normal cone to $K_{\Phi}$ at $\Phi\left(\bar{y}_{0}, \bar{y}_{T}\right)$, i.e.

$$
N_{K_{\Phi}}\left(\Phi\left(\bar{y}_{0}, \bar{y}_{T}\right)\right):=\left\{\Psi \in \mathbb{R}^{n_{\Phi} *}: \begin{array}{l}
\Psi_{i} \geq 0 \\
\Psi_{i} \Phi_{i}\left(\bar{y}_{0}, \bar{y}_{T}\right)=0
\end{array} \text { for } n_{\Phi^{E}}<i \leq n_{\Phi}\right\} .
$$

Finally, let

$$
N(\bar{u}, \bar{y}):=\mathbb{R}_{+} \times N_{K_{\Phi}}\left(\Phi\left(\bar{y}_{0}, \bar{y}_{T}\right)\right) \times N_{K_{c}}(c(\cdot, \bar{u}, \bar{y})) \times N_{K_{g}}(g(\cdot, \bar{y})) \subset E .
$$

We denote

$$
\mathcal{P}:=B V\left([0, T] ; \mathbb{R}^{n *}\right) .
$$

Given $(\bar{u}, \bar{y}) \in F(P)$ and $\lambda=(\beta, \Psi, \nu, \mu) \in E$, we consider the costate equation in $\mathcal{P}$

$$
\left\{\begin{aligned}
-\mathrm{d} p_{t} & =D_{y} H^{a}\left[p_{t}, \nu_{t}\right]\left(t, \bar{u}_{t}, \bar{y}_{t}\right) \mathrm{d} t+\mathrm{d} \mu_{t} D g\left(t, \bar{y}_{t}\right) \\
p_{T} & =D_{y_{T}} \Phi[\beta, \Psi]\left(\bar{y}_{0}, \bar{y}_{T}\right) .
\end{aligned}\right.
$$

Lemma 2.3. Let $(\bar{u}, \bar{y}) \in F(P)$. For any $\lambda \in E$, there exists a unique solution of the costate equation (2.17), that we denote by $p^{\lambda}$. The mapping

$$
\lambda \in E \mapsto p^{\lambda} \in \mathcal{P}
$$

is linear continuous.

Proof. We first get the existence, uniqueness and the continuity of

$$
\lambda \mapsto p^{\lambda} \in L^{1}\left(0, T ; \mathbb{R}^{n *}\right)
$$

by a contraction argument. Then the continuity of

$$
\lambda \mapsto\left(\mathrm{d} p, p_{T}\right) \in \mathcal{M}\left([0, T] ; \mathbb{R}^{n *}\right) \times \mathbb{R}^{n *}
$$

follows by (2.17).

Definition 2.4. Let $(\bar{u}, \bar{y}) \in F(P)$ and $\lambda=(\beta, \Psi, \nu, \mu) \in E$. We say that the solution of the costate equation (2.17) $p^{\lambda} \in \mathcal{P}$ is an associated costate iff

$$
-p_{0}^{\lambda}=D_{y_{0}} \Phi[\beta, \Psi]\left(\bar{y}_{0}, \bar{y}_{T}\right) .
$$

Let $N_{\pi}(\bar{u}, \bar{y})$ be the set of nonzero $\lambda \in N(\bar{u}, \bar{y})$ having an associated costate.

Let $(\bar{u}, \bar{y}) \in F(P)$. We define the set-valued mapping $U:[0, T] \rightrightarrows \mathbb{R}^{m}$ by

$$
U(t):=\operatorname{cl}\left\{u \in \mathbb{R}^{m}: c\left(t, u, \bar{y}_{t}\right)<0\right\} \quad \text { for a.a. } t,
$$

where cl denotes the closure in $\mathbb{R}^{m}$. 
Definition 2.5. Let $(\bar{u}, \bar{y}) \in F(P)$. We say that the inward condition for the mixed constraints holds iff there exist $\gamma>0$ and $\bar{v} \in \mathcal{U}$ such that

$$
c\left(t, \bar{u}_{t}, \bar{y}_{t}\right)+D_{u} c\left(t, \bar{u}_{t}, \bar{y}_{t}\right) \bar{v}_{t} \leq-\gamma, \quad \text { for a.a. } t .
$$

Remark 2.6. If the inward condition holds, then there exists $\delta>0$ such that, for a.a. $t$,

$$
B_{\delta}\left(\bar{u}_{t}\right) \cap U(t)=B_{\delta}\left(\bar{u}_{t}\right) \cap\left\{u \in \mathbb{R}^{m}: c\left(t, u, \bar{y}_{t}\right) \leq 0\right\},
$$

where $B_{\delta}\left(\bar{u}_{t}\right)$ is the open ball in $\mathbb{R}^{m}$ of center $\bar{u}_{t}$ and radius $\delta$. In particular, $\bar{u}_{t} \in U(t)$ for a.a. $t$.

In the sequel, we will always make the following assumption:

Assumption 2. The inward condition for the mixed constraints holds.

We can now define the notions of multipliers that we will consider. Recall that $N_{\pi}(\bar{u}, \bar{y})$ has been introduced in Definition 2.4

Definition 2.7. Let $(\bar{u}, \bar{y}) \in F(P)$.

(i) We say that $\lambda \in N_{\pi}(\bar{u}, \bar{y})$ is a generalized Lagrange multiplier iff

$$
D_{u} H^{a}\left[p_{t}^{\lambda}, \nu_{t}\right]\left(t, \bar{u}_{t}, \bar{y}_{t}\right)=0 \text { for a.a. } t .
$$

We denote by $\Lambda_{L}(\bar{u}, \bar{y})$ the set of generalized Lagrange multipliers.

(ii) We say that $\lambda \in \Lambda_{L}(\bar{u}, \bar{y})$ is a generalized Pontryagin multiplier iff

$$
H\left[p_{t}^{\lambda}\right]\left(t, \bar{u}_{t}, \bar{y}_{t}\right) \leq H\left[p_{t}^{\lambda}\right]\left(t, u, \bar{y}_{t}\right) \quad \text { for all } u \in U(t), \quad \text { for a.a. } t .
$$

We denote by $\Lambda_{P}(\bar{u}, \bar{y})$ the set of generalized Pontryagin multipliers.

(iii) We say that $\lambda \in \Lambda_{P}(\bar{u}, \bar{y})$ is a degenerate Pontryagin equality multiplier iff $\lambda=(\beta, \Psi, \nu, \mu)$ with $\Psi=\left(\Psi^{E}, \Psi^{I}\right)$ is such that $\left(\beta, \Psi^{I}, \nu, \mu\right)=0$ and if equality holds in $(2.26)$. We denote by $\Lambda_{P}^{D}(\bar{u}, \bar{y})$ the set of such multipliers.

Remark 2.8. 1. The sets $\Lambda_{L}(\bar{u}, \bar{y}), \Lambda_{P}(\bar{u}, \bar{y})$ and $\Lambda_{P}^{D}(\bar{u}, \bar{y})$ are positive cones of nonzero elements, possibly empty, and $\Lambda_{P}^{D}(\bar{u}, \bar{y})$ is symmetric.

2. Assumption 2 will be needed to get that the component $\nu$ of a multiplier, associated to the mixed constraints, belongs to $L^{\infty}\left(0, T ; \mathbb{R}^{n_{c} *}\right)$ and not only to $L^{\infty}\left(0, T ; \mathbb{R}^{n_{c}}\right)^{*}$. See $[7$, Theorem 3.1] and Theorem A.4 in Appendix A.1

3. Let $\lambda \in \Lambda_{P}(\bar{u}, \bar{y})$. If Assumption 2 holds, then by Remark 2.6, $\bar{u}_{t}$ is a local solution of the finite dimensional optimization problem

$$
\min _{u \in \mathbb{R}^{m}} H\left[p_{t}^{\lambda}\right]\left(t, u, \bar{y}_{t}\right) \quad \text { subject to } \quad c\left(t, u, \bar{y}_{t}\right) \leq 0,
$$

and $\nu_{t}$ is an associated Lagrange multiplier, for a.a. $t$.

4. See Appendix A.4 for an example where there exists a multiplier such that $(2.26)$ holds for all $u \in U(t)$, but not for all $u \in\left\{u \in \mathbb{R}^{m}: c\left(t, u, \bar{y}_{t}\right) \leq 0\right\}$.

We finish this section with various notions of minima, following [24]. 
Definition 2.9. We say that $(\bar{u}, \bar{y}) \in F(P)$ is a global minimum iff

$$
\phi\left(\bar{y}_{0}, \bar{y}_{T}\right) \leq \phi\left(y_{0}, y_{T}\right) \quad \text { for all }(u, y) \in F(P)
$$

a Pontryagin minimum iff for any $R>\|\bar{u}\|_{\infty}$, there exists $\varepsilon>0$ such that

$$
\begin{array}{ll}
\phi\left(\bar{y}_{0}, \bar{y}_{T}\right) \leq \phi\left(y_{0}, y_{T}\right) \quad & \text { for all }(u, y) \in F(P) \text { such that } \\
& \|u-\bar{u}\|_{1}+\|y-\bar{y}\|_{\infty} \leq \varepsilon \text { and }\|u\|_{\infty} \leq R,
\end{array}
$$

a weak minimum iff there exists $\varepsilon>0$ such that

$$
\begin{aligned}
\phi\left(\bar{y}_{0}, \bar{y}_{T}\right) \leq \phi\left(y_{0}, y_{T}\right) \quad & \text { for all }(u, y) \in F(P) \text { such that } \\
& \|u-\bar{u}\|_{\infty}+\|y-\bar{y}\|_{\infty} \leq \varepsilon .
\end{aligned}
$$

Remark 2.10. Obviously, $2.28 \Rightarrow 2.29 \Rightarrow 2.30$. Conversely, if $(\bar{u}, \bar{y})$ is a weak minimum for problem $(P)$, then it is a Pontryagin minimum for the problem obtained by adding the control constraint $\left|u_{t}-\bar{u}_{t}\right| \leq \varepsilon$, and a global minimum for the problem obtained by adding the same control constraint and the state constraint $\left|y_{t}-\bar{y}_{t}\right| \leq \varepsilon$.

\section{First-order conditions in Pontryagin form}

\subsection{Pontryagin's minimum principle}

First-order necessary conditions in Pontryagin form consist in proving the existence of Pontryagin multipliers. See Definitions 2.7 and 2.9 for the notions of multipliers and of minima. Our version of the well-known Pontryagin's principle follows, and is proved in Section 3.3. See [12, for a variant with the same approach, and [18] for a survey of this principle.

Theorem 3.1. Let $(\bar{u}, \bar{y})$ be a Pontryagin minimum for problem $(P$ and let Assumptions 1.2 hold. Then the set of generalized Pontryagin multipliers $\Lambda_{P}(\bar{u}, \bar{y})$ is nonempty.

By Remark 2.10, we get the following:

Corollary 3.2. Let $(\bar{u}, \bar{y})$ be a weak minimum for problem $(P$ and let Assumptions 1 . 2 hold. Then there exist $\varepsilon>0$ and $\lambda \in \Lambda_{L}(\bar{u}, \bar{y})$ such that

$$
\left\{\begin{array}{l}
\text { for a.a. } t, \text { for all } u \in U(t) \text { such that }\left|u-\bar{u}_{t}\right| \leq \varepsilon \\
H\left[p_{t}^{\lambda}\right]\left(t, \bar{u}_{t}, \bar{y}_{t}\right) \leq H\left[p_{t}^{\lambda}\right]\left(t, u, \bar{y}_{t}\right) .
\end{array}\right.
$$

Proof. The extra control constraint $\left|u-\bar{u}_{t}\right| \leq \varepsilon$ for a.a. $t$ is never active, therefore the set of Lagrange multipliers is unchanged. The set of Pontryagin multipliers is the set of Lagrange multipliers for which (3.1) holds.

The proof of Theorem 3.1, given in Section 3.3, relies on first-order necessary conditions for a family of weak minima for auxiliary optimal control problems, namely the partially relaxed problems, presented in Section 3.2. These problems are defined using a Castaing representation of the set-valued mapping $U$, introduced at the beginning of Section 3.2. Second order necessary conditions in Pontryagin form in Section 4.1 will be derived from a variant of the partially relaxed problems, the reduced partially relaxed problems. Thus Section 3.2 is central. First and second order necessary conditions for a weak minimum are recalled, with some orginal results, in Appendix A.1. 


\subsection{Partial relaxation}

In this section, $(\bar{u}, \bar{y})$ is a given Pontryagin minimum for problem $(\sqrt{P})$, and Assumptions 1 2 hold.

\subsubsection{Castaing representation}

See [9, 10, 28, for a general presentation of set-valued mappings and measurable selection theorems.

Definition 3.3. Let $V:[0, T] \rightrightarrows \mathbb{R}^{m}$ be a set-valued mapping. We say that a sequence $\left(v^{k}\right)_{k \in \mathbb{N}}$, $v^{k} \in \mathcal{U}$, is a Castaing representation of $V$ iff $\left\{v_{t}^{k}\right\}_{k \in \mathbb{N}}$ is a dense subset of $V(t)$ for a.a. $t$.

Lemma 3.4. There exists a Castaing representation $\left(u^{k}\right)_{k \in \mathbb{N}}$ of the set-valued mapping $U$ defined by (2.22), and for all $k$, there exists $\gamma_{k}>0$ such that

$$
c\left(t, u_{t}^{k}, \bar{y}_{t}\right) \leq-\gamma_{k} \quad \text { for a.a. } t .
$$

Proof. For $l \in \mathbb{N}, l \geq 1$, we consider the set-valued mapping $U_{l}$ defined by

$$
U_{l}(t):=\left\{u \in \mathbb{R}^{n}: c\left(t, u, \bar{y}_{t}\right) \leq-\frac{1}{l}\right\} \quad \text { for a.a. } t
$$

so that

$$
U(t)=\operatorname{cl}\left(\cup_{l \geq 1} U_{l}(t)\right) \quad \text { for a.a. } t \text {. }
$$

Under Assumptions 1, 2, by [9, Théorème 3.5] and for $l$ large enough, $U_{l}$ is a measurable with nonempty closed set-valued mapping. Then by [9, Théorème 5.4], it has a Castaing representation. By (3.4), the union of such Castaing representations for $l$ large enough is a Castaing representation of $U$.

We define the following sequence of sets of generalized Lagrange multipliers: for $N \in \mathbb{N}$, let

$$
\Lambda^{N}(\bar{u}, \bar{y}):=\left\{\lambda \in \Lambda_{L}(\bar{u}, \bar{y}): \begin{array}{l}
H\left[p_{t}^{\lambda}\right]\left(t, \bar{u}_{t}, \bar{y}_{t}\right) \leq H\left[p_{t}^{\lambda}\right]\left(t, u_{t}^{k}, \bar{y}_{t}\right) \\
\text { for all } k \leq N, \text { for a.a. } t
\end{array}\right\} .
$$

Observe that

$$
\Lambda_{P}(\bar{u}, \bar{y}) \subset \Lambda^{N+1}(\bar{u}, \bar{y}) \subset \Lambda^{N}(\bar{u}, \bar{y}) \subset \Lambda_{L}(\bar{u}, \bar{y}),
$$

and by density of the Castaing representation,

$$
\Lambda_{P}(\bar{u}, \bar{y})=\bigcap_{N \in \mathbb{N}} \Lambda^{N}(\bar{u}, \bar{y}) .
$$

Recall that $E$ and $\|\cdot\|_{E}$ have been defined by (2.10) and (2.11).

Lemma 3.5. Let $\left(\lambda^{N}\right)_{N \in \mathbb{N}}$ be a sequence in $\Lambda_{L}(\bar{u}, \bar{y})$ such that $\left\|\lambda^{N}\right\|_{E}=1$ and $\lambda^{N} \in \Lambda^{N}(\bar{u}, \bar{y})$ for all $N$. Then the sequence has at least one nonzero weak $*$ limit point that belongs to $\Lambda_{P}(\bar{u}, \bar{y})$.

Proof. By Assumption 2 and [7, Theorem 3.1], the sequence is bounded in $E$ for the usual norm, i.e. with $\|\nu\|_{\infty}$ instead of $\|\nu\|_{1}$. Then there exists $\bar{\lambda}$ such that, extracting a subsequence if necessary, $\lambda^{N} \rightarrow \bar{\lambda}$ for the weak $*$ topology. Since $N(\bar{u}, \bar{y})$ is weakly $*$ closed, $\bar{\lambda} \in N(\bar{u}, \bar{y})$. Observe now that if $\lambda \in N(\bar{u}, \bar{y})$, then

$$
\|\lambda\|_{E}=\beta+|\Psi|+\langle\nu, 1\rangle_{1}+\langle\mu, 1\rangle_{C}
$$

where $\langle\cdot, \cdot\rangle_{1}$ and $\langle\cdot, \cdot\rangle_{C}$ are the dual products in $L^{1}\left(0, T ; \mathbb{R}^{n_{c}}\right)$ and $C\left([0, T] ; \mathbb{R}^{n_{g}}\right)$, respectively, and the 1 are constant functions of appropriate size. Then $\|\bar{\lambda}\|=1$ and $\bar{\lambda} \neq 0$. Let $p^{N}:=p^{\lambda^{N}}$, 
$N \in \mathbb{N}$, and $\bar{p}:=p^{\bar{\lambda}}$. By Lemma $2.3, \mathrm{~d} p^{N} \rightarrow \mathrm{d} \bar{p}$ for the weak $*$ topology in $\mathcal{M}\left([0, T] ; \mathbb{R}^{n *}\right)$ and $p_{T}^{N} \rightarrow \bar{p}_{T}$. Since

$$
p_{0}=p_{T}-\langle\mathrm{d} p, 1\rangle_{C}
$$

for any $p \in \mathcal{P}$, we derive that $\bar{p}_{0}=D_{y_{0}} \Phi[\bar{\beta}, \bar{\Psi}]\left(\bar{y}_{0}, \bar{y}_{T}\right)$. Then $\bar{p}$ is an associated costate, i.e. $\bar{\lambda} \in N_{\pi}(\bar{u}, \bar{y})$. Next, as a consequence of Lemma 2.3 $p^{N} \rightarrow \bar{p}$ for the weak $*$ topology in $L^{\infty}$. Then $D_{u} H^{a}\left[p^{N}, \nu^{N}\right](\cdot, \bar{u}, \bar{y}) \rightarrow D_{u} H^{a}[\bar{p}, \bar{\nu}](\cdot, \bar{u}, \bar{y})$ for the weak $*$ topology in $L^{\infty}$, and then $D_{u} H^{a}\left[\bar{p}_{t}, \bar{\nu}_{t}\right]\left(t, \bar{u}_{t}, \bar{y}_{t}\right)=0$ for a.a. $t$, i.e. $\bar{\lambda} \in \Lambda_{L}(\bar{u}, \bar{y})$. Similarly, for all $k \in \mathbb{N}$,

$$
H\left[p^{N}\right]\left(\cdot, u^{k}, \bar{y}\right)-H\left[p^{N}\right](\cdot, \bar{u}, \bar{y}) \rightarrow H[\bar{p}]\left(\cdot, u^{k}, \bar{y}\right)-H[\bar{p}](\cdot, \bar{u}, \bar{y})
$$

for the weak $*$ topology in $L^{\infty}$, and then

$$
H\left[\bar{p}_{t}\right]\left(t, u_{t}^{k}, \bar{y}_{t}\right)-H\left[\bar{p}_{t}\right]\left(t, \bar{u}_{t}, \bar{y}_{t}\right) \geq 0 \text { for a.a. } t,
$$

i.e. $\bar{\lambda} \in \Lambda^{k}(\bar{u}, \bar{y})$, for all $k \in \mathbb{N}$. By $(3.7), \bar{\lambda} \in \Lambda_{P}(\bar{u}, \bar{y})$.

Since $\Lambda^{N}(\bar{u}, \bar{y}), N \in \mathbb{N}$, are cones of nonzero elements (see Remark 2.8), it is enough to show that they are nonempty for all $N$ to prove Theorem 3.1 by Lemma 3.5. This is the purpose of the partially relaxed problems, presented in the next section. Indeed, we will see that they are such that their Lagrange multipliers, whose existence can easily be guaranteed, belong to $\Lambda^{N}(\bar{u}, \bar{y})$.

\subsubsection{The partially relaxed problems}

As motivated above, we introduce now a sequence of optimal control problems.

Formulation Recall that $(\bar{u}, \bar{y})$ is given as a Pontryagin minimum for problem $(P)$ has been given.

Let $N \in \mathbb{N}$. Consider the partially relaxed state equation

$$
\dot{y}_{t}=\left(1-\sum_{i=1}^{N} \alpha_{t}^{i}\right) f\left(t, u_{t}, y_{t}\right)+\sum_{i=1}^{N} \alpha_{t}^{i} f\left(t, u_{t}^{i}, y_{t}\right) \quad \text { for a.a. } t \in(0, T) .
$$

The $u^{i}$ are elements of the Castaing representation given by Lemma 3.4. The controls are $u$ and $\alpha$, the state is $y$, with

$$
u \in \mathcal{U}, \quad \alpha \in \mathcal{A}^{N}:=L^{\infty}\left(0, T ; \mathbb{R}^{N}\right), \quad y \in \mathcal{Y} .
$$

The idea is to consider the problem of minimizing $\phi\left(y_{0}, y_{T}\right)$ under the same constraints as before, plus the control constraints $\alpha \geq 0$. To simplify the qualification issue, we actually introduce a slack variable $\theta \in \mathbb{R}$, with the intention to minimize it, and the following constraint on the cost function:

$$
\phi\left(y_{0}, y_{T}\right)-\phi\left(\bar{y}_{0}, \bar{y}_{T}\right) \leq \theta \text {. }
$$

The slack variable $\theta$ also enters into every inequality constraint:

$$
\begin{aligned}
-\alpha_{t} \leq \theta & \text { for a.a. } t \in(0, T), \\
c\left(t, u_{t}, y_{t}\right) \leq \theta & \text { for a.a. } t \in(0, T), \\
g\left(t, y_{t}\right) \leq \theta & \text { for a.a. } t \in(0, T), \\
\Phi^{I}\left(y_{0}, y_{T}\right) \leq \theta &
\end{aligned}
$$


and the equality constraints remain unchanged:

$$
\Phi^{E}\left(y_{0}, y_{T}\right)=0
$$

The partially relaxed problem is

$$
\min _{(u, \alpha, y, \theta) \in \mathcal{U} \times \mathcal{A}^{N} \times \mathcal{Y} \times \mathbb{R}} \theta \quad \text { subject to } 3.12-3.19 .
$$

Let $\bar{\alpha}:=0 \in \mathcal{A}^{N}$ and $\bar{\theta}:=0 \in \mathbb{R}$. As for problem $(P)$, we call a relaxed trajectory any $(u, \alpha, y, \theta)$ such that 3.12 holds. We say that a relaxed trajectory is feasible if it satisties constraints (3.14)-(3.19), and denote by $F\left(P_{N}\right)$ the set of feasible relaxed trajectories.

Under Assumption 1 for any $\left(u, \alpha, y^{0}\right) \in \mathcal{U} \times \mathcal{A}^{N} \times \mathbb{R}^{n}$, there exists a unique $y \in \mathcal{Y}$ such that (3.12 holds and $y_{0}=y^{0}$; we denote it by $y\left[u, \alpha, y^{0}\right]$ and consider the mapping

$$
\Gamma_{N}:\left(u, \alpha, y^{0}\right) \mapsto y\left[u, \alpha, y^{0}\right]
$$

Remark 3.6. 1. We have $(\bar{u}, \bar{\alpha}, \bar{y}, \bar{\theta}) \in F\left(P_{N}\right)$.

2. Robinson's constraint qualification holds at $(\bar{u}, \bar{\alpha}, \bar{y}, \bar{\theta})$ iff the equality constraints are qualified, i.e. iff the derivative of

$$
\left(u, \alpha, y^{0}\right) \in \mathcal{U} \times \mathcal{A}^{N} \times \mathbb{R}^{n} \mapsto \Phi^{E}\left(y^{0}, \Gamma_{N}\left(u, \alpha, y^{0}\right)_{T}\right) \in \mathbb{R}^{n_{\Phi} E}
$$

at $\left(\bar{u}, \bar{\alpha}, \bar{y}_{0}\right)$ is onto. We say that problem $\left(P_{N}\right)$ is qualified at $(\bar{u}, \bar{\alpha}, \bar{y}, \bar{\theta})$ if this is the case. See [8, Section 2.3.4] for the definition and characterizations of Robinson's constraint qualification.

Existence of a minimum A key result is the following:

Theorem 3.7. Let Assumptions 1 2 hold and let problem $\sqrt{P_{N}}$ be qualified at $(\bar{u}, \bar{\alpha}, \bar{y}, \bar{\theta})$. Then $(\bar{u}, \bar{\alpha}, \bar{y}, \bar{\theta})$ is a weak minimum for this problem.

Theorem 3.7 is a corollary of the following proposition, proved in the Appendix A.2 for the sake of self-containment of the paper. It can also be deduced from other classical relaxation theorems, such as [13, Theorem 3].

Proposition 3.8. Under the assumptions of Theorem 3.7 there exists $M>0$ such that, for any $(\hat{u}, \hat{\alpha}, \hat{y}, \hat{\theta}) \in F\left(P_{N}\right)$ in a $L^{\infty}$ neighborhood of $(\bar{u}, \bar{\alpha}, \bar{y}, \bar{\theta})$ and with $\hat{\theta}<0$, for any $\varepsilon>0$, there exists $(\tilde{u}, \tilde{y}) \in F(P)$ such that

$$
\|\tilde{u}-\hat{u}\|_{1} \leq M\|\hat{\alpha}\|_{\infty} \quad \text { and } \quad\|\tilde{y}-\hat{y}\|_{\infty} \leq \varepsilon .
$$

Proof of Theorem 3.7. Suppose that $(\bar{u}, \bar{\alpha}, \bar{y}, \bar{\theta})$ is not a weak minimum for problem $\left(P_{N}\right)$. Then there exists $(\hat{u}, \hat{\alpha}, \hat{y}, \hat{\theta}) \in F\left(P_{N}\right)$ as $L^{\infty}$ close to $(\bar{u}, \bar{\alpha}, \bar{y}, \bar{\theta})$ as needed and with $\hat{\theta}<0$. Let $\varepsilon>0$ be such that

$$
\|y-\hat{y}\|_{\infty} \leq \varepsilon \Rightarrow \phi\left(y_{0}, y_{T}\right)<\phi\left(\bar{y}_{0}, \bar{y}_{T}\right) .
$$

By the proposition, we get $(\tilde{u}, \tilde{y}) \in F(P)$ such that $\phi\left(\tilde{y}_{0}, \tilde{y}_{T}\right)<\phi\left(\bar{y}_{0}, \bar{y}_{T}\right)$ and

$$
\|\tilde{u}-\bar{u}\|_{1}+\|\tilde{y}-\bar{y}\|_{\infty} \leq M\|\hat{\alpha}\|_{\infty}+T\|\hat{u}-\bar{u}\|_{\infty}+\varepsilon+\|\hat{y}-\bar{y}\|_{\infty} .
$$

Observe that the right-hand side of $(3.24)$ can be chosen as small as needed. Thus we get a contradiction with the Pontryagin optimality of $(\bar{u}, \bar{y})$.

$\mathrm{RR} \mathrm{n}^{\circ} 8306$ 
Optimality conditions Problem $\sqrt{P_{N}}$ can be seen as an optimization problem over $\left(u, \alpha, y^{0}, \theta\right) \in$ $\mathcal{U} \times \mathcal{A}^{N} \times \mathbb{R}^{n} \times \mathbb{R}$, via the mapping $\Gamma_{N}$ defined by $(3.20)$. Then we can define the set $\Lambda\left(P_{N}\right)$ of Lagrange multipliers at $\left(\bar{u}, \bar{\alpha}, \bar{y}_{0}, \bar{\theta}\right)$ as in Appendix $\bar{A} .1$

$$
\Lambda\left(P_{N}\right):=\left\{(\lambda, \gamma) \in N(\bar{u}, \bar{y}) \times L^{\infty}\left(0, T ; \mathbb{R}_{+}^{N *}\right): D L_{N}[\lambda, \gamma]\left(\bar{u}, \bar{\alpha}, \bar{y}_{0}, \bar{\theta}\right)=0\right\}
$$

where $L_{N}$ is defined, for $\lambda=(\beta, \Psi, \nu, \mu), \Psi=\left(\Psi^{E}, \Psi^{I}\right), y=\Gamma_{N}\left(u, \alpha, y^{0}\right)$, by

$$
\begin{aligned}
L_{N}[\lambda, \gamma]\left(u, \alpha, y^{0}, \theta\right):= & \theta+\beta\left(\phi\left(y_{0}, y_{T}\right)-\phi\left(\bar{y}_{0}, \bar{y}_{T}\right)-\theta\right) \\
& \quad+\Psi^{E} \Phi^{E}\left(y_{0}, y_{T}\right)+\Psi^{I}\left(\Phi^{I}\left(y_{0}, y_{T}\right)-\theta\right) \\
& +\int_{[0, T]}\left[\nu_{t}\left(c\left(t, u_{t}, y_{t}\right)-\theta\right) \mathrm{d} t+\mathrm{d} \mu_{t}\left(g\left(t, y_{t}\right)-\theta\right)-\gamma_{t}\left(\alpha_{t}+\theta\right) \mathrm{d} t\right] .
\end{aligned}
$$

In $3.26, \theta$ has to be understood as a vector of appropriate size and with equal components. We have the following first-order necessary conditions:

Lemma 3.9. Let problem $\sqrt{P_{N}}$ be qualified at $(\bar{u}, \bar{\alpha}, \bar{y}, \bar{\theta})$. Then $\Lambda\left(P_{N}\right)$ is nonempty, convex, and weakly $*$ compact.

Proof. We apply Theorem A.4 to $\left(\bar{u}, \bar{\alpha}, \bar{y}_{0}, \bar{\theta}\right)$, locally optimal solution of $\left(P_{N}\right)$ by Theorem 3.7 . Let $\bar{v} \in \mathcal{U}$ be given by the inward condition for the mixed constraints in problem $(P)$ (Assumption 2 and let $\bar{\omega}:=1 \in \mathcal{A}^{N}$. Then $(\bar{v}, \bar{\omega})$ satisfies the inward condition for the mixed constraints in problem $\left(P_{N}\right)$. The other assumptions being also satisfied by Assumption 1 and Remark 3.6 , the conclusion follows.

\subsection{Proof of Theorem 3.1}

As explained at the end of Section 3.2.1 it is enough by Lemma 3.5 to prove that $\Lambda^{N}(\bar{u}, \bar{y}) \neq \emptyset$ for all $N$. To do so, we use the partially relaxed problems $\left(P_{N}\right)$ as follows:

Lemma 3.10. Let $(\lambda, \gamma) \in \Lambda\left(P_{N}\right)$. Then $\lambda \in \Lambda^{N}(\bar{u}, \bar{y})$.

Proof. Let $(u, \alpha, y, \theta)$ be a relaxed trajectory and $(\lambda, \gamma) \in E \times L^{\infty}\left(0, T ; \mathbb{R}^{N *}\right)$, with $\lambda=(\beta, \Psi, \nu, \mu)$ and $\Psi=\left(\Psi^{E}, \Psi^{I}\right)$. Adding to $L_{N}$

$$
0=\int_{0}^{T} p_{t}\left(\left(1-\sum \alpha_{t}^{i}\right) f\left(t, u_{t}, y_{t}\right)+\sum \alpha_{t}^{i} f\left(t, u_{t}^{i}, y_{t}\right)-\dot{y}_{t}\right) \mathrm{d} t,
$$

and integrating by parts we have, for any $p \in \mathcal{P}$,

$$
\begin{aligned}
& L_{N}[\lambda, \gamma]\left(u, \alpha, y_{0}, \theta\right)=\theta\left(1-\beta-\left\langle\Psi^{I}, 1\right\rangle-\langle\nu, 1\rangle_{1}-\langle\mu, 1\rangle_{C}-\langle\gamma, 1\rangle_{1}\right) \\
& \quad+\int_{0}^{T}\left(H^{a}\left[p_{t}, \nu_{t}\right]\left(t, u_{t}, y_{t}\right)+\sum_{i=1}^{N} \alpha_{t}^{i}\left(H\left[p_{t}\right]\left(t, u_{t}^{i}, y_{t}\right)-H\left[p_{t}\right]\left(t, u_{t}, y_{t}\right)-\gamma_{t}^{i}\right)\right) \mathrm{d} t \\
& \quad+\int_{[0, T]}\left(\mathrm{d} \mu_{t} g\left(t, y_{t}\right)+\mathrm{d} p_{t} y_{t}\right)+\Phi[\beta, \Psi]\left(y_{0}, y_{T}\right)-p_{T} y_{T}+p_{0} y_{0}-\beta \phi\left(\bar{y}_{0}, \bar{y}_{T}\right) .
\end{aligned}
$$

Let $(\lambda, \gamma) \in \Lambda\left(P_{N}\right)$. Using the expression 3.28 of $L_{N}$, we get

$$
\begin{aligned}
& D_{y_{0}} \Phi[\beta, \Psi]\left(\bar{y}_{0}, \bar{y}_{T}\right)+p_{0}^{\lambda}=0, \\
& D_{u} H^{a}\left[p_{t}^{\lambda}, \nu_{t}\right]\left(t, \bar{u}_{t}, \bar{y}_{t}\right)=0 \text { for a.a. } t, \\
& H\left[p_{t}^{\lambda}\right]\left(t, u_{t}^{i}, \bar{y}_{t}\right)-H\left[p_{t}^{\lambda}\right]\left(t, \bar{u}_{t}, \bar{y}_{t}\right)=\gamma_{t}^{i} \text { for a.a. } t, \quad 1 \leq i \leq N, \\
& \beta+\left\langle\Psi^{I}, 1\right\rangle+\langle\nu, 1\rangle_{1}+\langle\mu, 1\rangle_{C}+\langle\gamma, 1\rangle_{1}=1 .
\end{aligned}
$$


Suppose that $\lambda=0$. Then $p^{\lambda}=0$ and by (3.31), $\gamma=0$; we get a contradiction with 3.32 . Then $\lambda \neq 0$ and $\lambda \in N_{\pi}(\bar{u}, \bar{y})$ by $(3.29)$. Finally, $\lambda \in \Lambda_{L}(\bar{u}, \bar{y})$ by $(3.30)$, and $\lambda \in \Lambda^{N}(\bar{u}, \bar{y})$ by (3.31) since $\gamma \in L^{\infty}\left(0, T ; \mathbb{R}_{+}^{N *}\right)$.

We need one more lemma:

Lemma 3.11. Let problem $\sqrt{P_{N}}$ be not qualified at $(\bar{u}, \bar{\alpha}, \bar{y}, \bar{\theta})$. Then there exists $\lambda \in \Lambda^{N}(\bar{u}, \bar{y})$ such that $-\lambda \in \Lambda^{N}(\bar{u}, \bar{y})$ too, and for all $k \leq N$,

$$
H\left[p_{t}^{\lambda}\right]\left(t, \bar{u}_{t}, \bar{y}_{t}\right)=H\left[p_{t}^{\lambda}\right]\left(t, u_{t}^{i}, \bar{y}_{t}\right) \quad \text { for a.a. } t .
$$

Proof. Recall that $\Gamma_{N}$ has been defined by 3.20 . By Remark 3.6 .2 , there exists $\Psi^{E} \neq 0$ such that

$$
\Psi^{E} D \Phi^{E}\left(\bar{y}_{0}, \bar{y}_{T}\right) D \Gamma_{N}\left(\bar{u}, \bar{\alpha}, \bar{y}_{0}\right)=0 .
$$

Let $\Psi=\left(\Psi^{E}, 0\right)$ and $\lambda:=(0, \Psi, 0,0)$, so that $D_{\left(u, \alpha, y^{0}\right)} L_{N}[\lambda, 0]\left(\bar{u}, \bar{\alpha}, \bar{y}_{0}, \bar{\theta}\right)=0$ by $(3.26)$. By (3.28), we get

$$
\begin{aligned}
& D_{y_{0}} \Phi\left[0,\left(\Psi^{E}, 0\right)\right]\left(\bar{y}_{0}, \bar{y}_{T}\right)+p_{0}^{\lambda}=0, \\
& D_{u} H^{a}\left[p_{t}^{\lambda}, 0\right]\left(t, \bar{u}_{t}, \bar{y}_{t}\right)=0 \text { for a.a. } t, \\
& H\left[p_{t}^{\lambda}\right]\left(t, u_{t}^{i}, \bar{y}_{t}\right)-H\left[p_{t}^{\lambda}\right]\left(t, \bar{u}_{t}, \bar{y}_{t}\right)=0 \text { for a.a. } t, \quad 1 \leq i \leq N .
\end{aligned}
$$

Then $\lambda \in \Lambda^{N}(\bar{u}, \bar{y})$ and $(3.33)$ holds.

We can now conclude:

Proof of Theorem 3.1. We need $\Lambda^{N}(\bar{u}, \bar{y}) \neq \emptyset$ for all $N$. If problem $\left(P_{N}\right)$ is qualified at $(\bar{u}, \bar{\alpha}, \bar{y}, \bar{\theta})$, then $\Lambda^{N}(\bar{u}, \bar{y}) \neq \emptyset$ by Lemmas 3.9 and 3.10 . If problem $\left(P_{N}\right)$ is not qualified at $(\bar{u}, \bar{\alpha}, \bar{y}, \bar{\theta})$, then $\Lambda^{N}(\bar{u}, \bar{y}) \neq \emptyset$ by Lemma 3.11 .

Actually, we have the following alternative:

Corollary 3.12. The partially relaxed problems $\left(P_{N}\right.$ are either qualified for all $N$ large enough, if $\Lambda_{P}^{D}(\bar{u}, \bar{y})=\emptyset$, or never qualified, and then $\Lambda_{P}^{D}(\bar{u}, \bar{y}) \neq \emptyset$.

Proof. If the problems $\left(P_{N}\right)$ are never qualified, then we get a sequence a multipliers as in the proof of Lemma 3.11. By the proof of Lemma 3.5 its limit points belong to $\Lambda_{P}^{D}(\bar{u}, \bar{y})$.

See Appendix A.3 for a qualification condition ensuring the non singularity of the generalized Pontryagin multipliers.

\section{Second-order conditions in Pontryagin form}

\subsection{Statement}

The second-order necessary conditions presented in this section involve Pontryagin multipliers only. They rely again on the partially relaxed problems, introduced in Section 3.2. These problems are actually modified into reduced partially relaxed problems, which satisfy an extended polyhedricity condition, [8, Section 3.2.3]. The idea is to get our second-order necessary conditions on a large cone by density of the so-called strict radial critical cone, so that we do not have to compute the envelope-like effect, Kawasaki [21.

The main result of this section is Theorem 4.9. It is stated after some new definitions and assumptions, and proved in Section 4.2

$\mathrm{RR} \mathrm{n}^{\circ} 8306$ 


\subsubsection{Definitions and assumptions}

For second-order optimality conditions, we need a stronger regularity assumption than Assumption 1 . Namely, we make in the sequel the following:

Assumption 3. The mappings $f$ and $g$ are $C^{\infty}, c$ is uniformly quasi- $C^{2}, \Phi$ and $\phi$ are $C^{2}$.

Remark 4.1. If there is no pure state constraint in problem $(P$ (i.e. no mapping $g$ ), we will see that it is enough to assume that $f$ is uniformly quasi- $C^{2}$.

For $s \in[1, \infty]$, let

$$
\mathcal{V}_{s}:=L^{s}\left(0, T ; \mathbb{R}^{m}\right), \quad \mathcal{Z}_{s}:=W^{1, s}\left(0, T ; \mathbb{R}^{n}\right)
$$

Let $(\bar{u}, \bar{y})$ be a trajectory for problem $(P)$. Given $v \in \mathcal{V}_{s}, s \in[1, \infty]$, we consider the linearized state equation in $\mathcal{Z}_{s}$

$$
\dot{z}_{t}=D f\left(t, \bar{u}_{t}, \bar{y}_{t}\right)\left(v_{t}, z_{t}\right) \quad \text { for a.a. } t \in(0, T) .
$$

We call a linearized trajectory any $(v, z) \in \mathcal{V}_{s} \times \mathcal{Z}_{s}$ such that 4.2 holds. For any $\left(v, z^{0}\right) \in \mathcal{V}_{s} \times \mathbb{R}^{n}$, there exists a unique $z \in \mathcal{Z}_{s}$ such that $(4.2)$ holds and $z_{0}=z^{0}$; we denote it by $z=z\left[v, z^{0}\right]$.

For $1 \leq i \leq n_{g}$, we define $g_{i}^{(j)}:[0, T] \times \mathbb{R}^{m} \times \mathbb{R}^{n} \rightarrow \mathbb{R}, j \in \mathbb{N}$, recursively by

$$
g_{i}^{(j+1)}(t, u, y):=D_{t} g_{i}^{(j)}(t, u, y)+D_{y} g_{i}^{(j)}(t, u, y) f(t, u, y), \quad g_{i}^{(0)}:=g_{i} .
$$

Definition 4.2. The order of a state constraint $g_{i}$ is $q_{i} \in \mathbb{N}$ such that

$$
D_{u} g_{i}^{(j)} \equiv 0 \quad \text { for } 0 \leq j \leq q_{i}-1, \quad D_{u} g_{i}^{\left(q_{i}\right)} \not \equiv 0 .
$$

Remark 4.3. If $g_{i}$ is of order $q_{i}$, then $t \mapsto g_{i}\left(t, \bar{y}_{t}\right) \in W^{q_{i}, \infty}(0, T)$ for any trajectory $(\bar{u}, \bar{y})$, and

$$
\begin{aligned}
\frac{\mathrm{d}^{j}}{\mathrm{~d} t^{j}} g_{i}\left(t, \bar{y}_{t}\right) & =g_{i}^{(j)}\left(t, \bar{y}_{t}\right) \quad \text { for } 0 \leq j \leq q_{i}-1, \\
\frac{\mathrm{d}^{q_{i}}}{\mathrm{~d} t^{q_{i}}} g_{i}\left(t, \bar{y}_{t}\right) & =g_{i}^{\left(q_{i}\right)}\left(t, \bar{u}_{t}, \bar{y}_{t}\right) .
\end{aligned}
$$

We have the same regularity along linearized trajectories; the proof of the next lemma is classical, see for instance [5, Lemma 9].

Lemma 4.4. Let $(\bar{u}, \bar{y})$ be a trajectory and $(v, z) \in \mathcal{V}_{s} \times \mathcal{Z}_{s}$ be a linearized trajectory, $s \in[1, \infty]$. Let the constraint $g_{i}$ be of order $q_{i}$. Then

$$
t \mapsto D g_{i}\left(t, \bar{y}_{t}\right) z_{t} \in W^{q_{i}, s}(0, T),
$$

and

$$
\begin{aligned}
\frac{\mathrm{d}^{j}}{\mathrm{~d} t^{j}} D g_{i}\left(t, \bar{y}_{t}\right) z_{t} & =D g_{i}^{(j)}\left(t, \bar{y}_{t}\right) z_{t} \quad \text { for } 0 \leq j \leq q_{i}-1, \\
\frac{\mathrm{d}^{q_{i}}}{\mathrm{~d} t^{q_{i}}} D g_{i}\left(t, \bar{y}_{t}\right) z_{t} & =D g_{i}^{\left(q_{i}\right)}\left(t, \bar{u}_{t}, \bar{y}_{t}\right)\left(v_{t}, z_{t}\right) .
\end{aligned}
$$

Definition 4.5. Let $(\bar{u}, \bar{y}) \in F(P)$. We say that $\tau \in[0, T]$ is a touch point for the constraint $g_{i}$ iff it is a contact point for $g_{i}$, i.e. $g_{i}\left(\tau, \bar{y}_{\tau}\right)=0$, isolated in $\left\{t: g_{i}\left(t, \bar{y}_{t}\right)=0\right\}$. We say that a touch point $\tau$ for $g_{i}$ is reducible iff $\tau \in(0, T), \frac{\mathrm{d}^{2}}{\mathrm{~d} t^{2}} g_{i}\left(t, \bar{y}_{t}\right)$ is defined for $t$ close to $\tau$, continuous at $\tau$, and

$$
\left.\frac{\mathrm{d}^{2}}{\mathrm{~d} t^{2}} g_{i}\left(t, \bar{y}_{t}\right)\right|_{t=\tau}<0
$$


Remark 4.6. If $g_{i}$ is of order at least 2, then by Remark 4.3 a touch point $\tau$ for $g_{i}$ is reducible iff $t \mapsto g_{i}^{(2)}\left(t, \bar{u}_{t}, \bar{y}_{t}\right)$ is continuous at $\tau$ and $g_{i}^{(2)}\left(\tau, \bar{u}_{\tau}, \bar{y}_{\tau}\right)<0$. The continuity holds if $\bar{u}$ is continuous at $\tau$ or if $g_{i}$ is of order at least 3 .

Let $(\bar{u}, \bar{y}) \in F(P)$. For $1 \leq i \leq n_{g}$, let

$$
\begin{aligned}
\mathcal{T}_{g, i} & := \begin{cases}\emptyset & \text { if } g_{i} \text { is of order } 1, \\
\left\{\text { touch points for } g_{i}\right\} & \text { if } g_{i} \text { is of order at least 2, }\end{cases} \\
\Delta_{g, i}^{0}: & =\left\{t \in[0, T]: g_{i}\left(t, \bar{y}_{t}\right)=0\right\} \backslash \mathcal{T}_{g, i}, \\
\Delta_{g, i}^{\varepsilon} & :=\left\{t \in[0, T]: \operatorname{dist}\left(t, \Delta_{g, i}^{0}\right) \leq \varepsilon\right\},
\end{aligned}
$$

and for $1 \leq i \leq n_{c}$, let

$$
\Delta_{c, i}^{\delta}:=\left\{t \in[0, T]: c_{i}\left(t, \bar{u}_{t}, \bar{y}_{t}\right) \geq-\delta\right\} .
$$

We will need the following two extra assumptions:

Assumption 4. For $1 \leq i \leq n_{g}$, the set $\mathcal{T}_{g, i}$ is finite and contains only reducible touch points, $\Delta_{g, i}^{0}$ has finitely many connected components and $g_{i}$ is of finite order $q_{i}$.

Assumption 5. There exist $\delta^{\prime}, \varepsilon^{\prime}>0$ such that the linear mapping from $\mathcal{V}_{2} \times \mathbb{R}^{n}$ to $\prod_{i=1}^{n_{c}} L^{2}\left(\Delta_{c, i}^{\delta^{\prime}}\right) \times$ $\prod_{i=1}^{n_{g}} W^{q_{i}, 2}\left(\Delta_{g, i}^{\varepsilon^{\prime}}\right)$ defined by

$$
\left(v, z^{0}\right) \mapsto\left(\begin{array}{c}
\left(\left.D c_{i}(\cdot, \bar{u}, \bar{y})\left(v, z\left[v, z^{0}\right]\right)\right|_{\Delta_{c, i}^{\delta^{\prime}}}\right)_{1 \leq i \leq n_{c}} \\
\left(\left.D g_{i}(\cdot, \bar{y}) z\left[v, z^{0}\right]\right|_{\Delta_{g, i}^{\varepsilon^{\prime}}}\right)_{1 \leq i \leq n_{g}}
\end{array}\right) \quad \text { is onto. }
$$

Remark 4.7. There exist sufficient conditions, of linear independance type, for Assumption 5 to hold. See for instance [6, Lemma 2.3] or [3, Lemma 4.5].

\subsubsection{Main result}

Let $(\bar{u}, \bar{y}) \in F(P)$. We define the critical cone in $L^{2}$

$$
C_{2}(\bar{u}, \bar{y}):=\left\{\begin{array}{l}
(v, z) \in \mathcal{V}_{2} \times \mathcal{Z}_{2}: z=z\left[v, z_{0}\right] \\
D \phi\left(\bar{y}_{0}, \bar{y}_{T}\right)\left(z_{0}, z_{T}\right) \leq 0 \\
D \Phi\left(\bar{y}_{0}, \bar{y}_{T}\right)\left(z_{0}, z_{T}\right) \in T_{K_{\Phi}}\left(\Phi\left(\bar{y}_{0}, \bar{y}_{T}\right)\right) \\
D c(\cdot, \bar{u}, \bar{y})(v, z) \in T_{K_{c}}(c(\cdot, \bar{u}, \bar{y})) \\
D g(\cdot, \bar{y}) z \in T_{K_{g}}(g(\cdot, \bar{y}))
\end{array}\right\}
$$

and the strict critical cone in $L^{2}$

$$
C_{2}^{S}(\bar{u}, \bar{y}):=\left\{\begin{array}{lll}
(v, z) \in C_{2}(\bar{u}, \bar{y}): & \\
D c_{i}\left(t, \bar{u}_{t}, \bar{y}_{t}\right)\left(v_{t}, z_{t}\right)=0 & t \in \Delta_{c, i}^{0} \quad 1 \leq i \leq n_{c} \\
D g_{i}\left(t, \bar{y}_{t}\right) z_{t}=0 & t \in \Delta_{g, i}^{0} \quad 1 \leq i \leq n_{g}
\end{array}\right\}
$$

Remark 4.8. 1. See [8, Examples 2.63 and 2.64] for the description of $T_{K_{g}}$ and $T_{K_{c}}$, respectively.

RR n 8306 
2. Since by Assumption 4 there are finitely many touch points for constraints of order at least $2, C_{2}^{S}(\bar{u}, \bar{y})$ is defined by equality constraints and a finite number of inequality constraints, i.e. the cone $C_{2}^{S}(\bar{u}, \bar{y})$ is a polyhedron.

3. The strict critical cone $C_{2}^{S}(\bar{u}, \bar{y})$ is a subset of the critical cone $C_{2}(\bar{u}, \bar{y})$. But if there exists $\lambda=(\bar{\beta}, \bar{\Psi}, \bar{\nu}, \bar{\mu}) \in \Lambda_{L}(\bar{u}, \bar{y})$ such that

$$
\begin{array}{ll}
\bar{\nu}_{i}(t)>0 \quad \text { for a.a. } t \in \Delta_{c, i}^{0} & 1 \leq i \leq n_{c}, \\
\Delta_{g, i}^{0} \subset \operatorname{supp}\left(\bar{\mu}_{i}\right) & 1 \leq i \leq n_{g}
\end{array}
$$

then $C_{2}^{S}(\bar{u}, \bar{y})=C_{2}(\bar{u}, \bar{y})$ (see [8, Proposition 3.10]).

For any $\lambda=(\beta, \Psi, \nu, \mu) \in E$, we define a quadratic form, the Hessian of Lagrangian, $\Omega[\lambda]: \mathcal{V}_{2} \times \mathcal{Z}_{2} \rightarrow \mathbb{R}$ by

$$
\begin{aligned}
\Omega[\lambda](v, z):=\int_{0}^{T} D^{2} H^{a}\left[p_{t}^{\lambda}, \nu_{t}\right]\left(t, \bar{u}_{t}, \bar{y}_{t}\right)\left(v_{t}, z_{t}\right)^{2} \mathrm{~d} t+D^{2} \Phi[\beta, \Psi]\left(\bar{y}_{0}, \bar{y}_{T}\right)\left(z_{0}, z_{T}\right)^{2} \\
+\int_{[0, T]} \mathrm{d} \mu_{t} D^{2} g\left(t, \bar{y}_{t}\right)\left(z_{t}\right)^{2}-\sum_{\substack{\tau \in \mathcal{T}_{g, i} \\
1 \leq i \leq n_{g}}} \mu_{i}(\tau) \frac{\left(D g_{i}^{(1)}\left(\tau, \bar{y}_{\tau}\right) z_{\tau}\right)^{2}}{g_{i}^{(2)}\left(\tau, \bar{u}_{\tau}, \bar{y}_{\tau}\right)}
\end{aligned}
$$

We can now state our main result, that will be proved in the next section.

Theorem 4.9. Let $(\bar{u}, \bar{y})$ be a Pontryagin minimum for problem $(P$ and let Assumptions 2 hold. Then for any $(v, z) \in C_{2}^{S}(\bar{u}, \bar{y})$, there exists $\lambda \in \Lambda_{P}(\bar{u}, \bar{y})$ such that

$$
\Omega[\lambda](v, z) \geq 0
$$

Remark 4.10. If $\Lambda_{P}^{D}(\bar{u}, \bar{y}) \neq \emptyset$ and $\lambda \in \Lambda_{P}^{D}(\bar{u}, \bar{y})$, then $-\lambda \in \Lambda_{P}^{D}(\bar{u}, \bar{y})$ too. Since $\Omega[-\lambda](v, z)=$ $-\Omega[\lambda](v, z)$ for any $(v, z) \in \mathcal{V}_{2} \times \mathcal{Z}_{2}$, Theorem 4.9 is then pointless. See Corollary 3.12 about the emptiness of $\Lambda_{P}^{D}(\bar{u}, \bar{y})$.

\subsection{Proof of Theorem 4.9}

In this section, $(\bar{u}, \bar{y})$ is a given Pontryagin minimum for problem $(P)$, and Assumptions 2,5 hold.

\subsubsection{Reduction and partial relaxation}

The reduction approach [5, section 5] consists in reformulating the state constraint in the neighborhood of a touch point, using its reducibility (Definition 4.5). We apply this approach to the partially relaxed problems $\left(P_{N}\right)$ in order to involve Pontryagin multipliers (see Lemmas 3.5 and 3.10 .

Let $N \in \mathbb{N}$. Recall that $\Gamma_{N}$ has been defined by $(3.20)$.

Remark 4.11. The result of Remark 4.3 still holds for relaxed trajectories:

$$
t \mapsto g_{i}\left(t, y_{t}\right) \in W^{q_{i}, \infty}(0, T) \text { for any } y=\Gamma_{N}\left(u, \alpha, y^{0}\right) .
$$

Let $\tau \in \mathcal{T}_{g, i}$. We define $\Theta_{i, \tau}^{\varepsilon, N}: \mathcal{U} \times \mathcal{A}^{N} \times \mathbb{R}^{n} \rightarrow \mathbb{R}$ by

$$
\Theta_{i, \tau}^{\varepsilon, N}\left(u, \alpha, y^{0}\right):=\max \left\{g_{i}\left(t, y_{t}\right): y=\Gamma_{N}\left(u, \alpha, y^{0}\right), t \in[\tau-\varepsilon, \tau+\varepsilon] \cap[0, T]\right\} .
$$

Let $\bar{\Gamma}_{N}^{\prime}:=D \Gamma_{N}\left(\bar{u}, \bar{\alpha}, \bar{y}_{0}\right)$ and $\bar{\Gamma}_{N}^{\prime \prime}:=D^{2} \Gamma_{N}\left(\bar{u}, \bar{\alpha}, \bar{y}_{0}\right)$. 
Remark 4.12. Let $\bar{\omega}:=0 \in \mathcal{A}^{N}$. For any $\left(v, z^{0}\right) \in \mathcal{V}_{s} \times \mathbb{R}^{n}, s \in[1, \infty]$, we have

$$
\bar{\Gamma}_{N}^{\prime}\left(v, \bar{\omega}, z^{0}\right)=z\left[v, z^{0}\right]
$$

Lemma 4.13. There exists $\varepsilon>0$ independent of $N$ such that for any $\tau \in \mathcal{T}_{g, i}, \Theta_{i, \tau}^{\varepsilon, N}$ is $C^{1}$ in a neighborhood of $\left(\bar{u}, \bar{\alpha}, \bar{y}_{0}\right)$ and twice Fréchet differentiable at $\left(\bar{u}, \bar{\alpha}, \bar{y}_{0}\right)$, with first and second derivatives given by

$$
D \Theta_{i, \tau}^{\varepsilon, N}\left(\bar{u}, \bar{\alpha}, \bar{y}_{0}\right)\left(v, \omega, z^{0}\right)=D g_{i}\left(\tau, \bar{y}_{\tau}\right) \bar{\Gamma}_{N}^{\prime}\left(v, \omega, z^{0}\right)_{\tau}
$$

for any $\left(v, \omega, z^{0}\right) \in \mathcal{V}_{1} \times L^{1}\left(0, T ; R^{N}\right) \times \mathbb{R}^{n}$, and

$$
\begin{aligned}
D^{2} \Theta_{i, \tau}^{\varepsilon, N}\left(\bar{u}, \bar{\alpha}, \bar{y}_{0}\right)\left(v, \omega, z^{0}\right)^{2}= & D^{2} g_{i}\left(\tau, \bar{y}_{\tau}\right)\left(\bar{\Gamma}_{N}^{\prime}\left(v, \omega, z^{0}\right)_{\tau}\right)^{2} \\
& +D g_{i}\left(\tau, \bar{y}_{\tau}\right) \bar{\Gamma}_{N}^{\prime \prime}\left(v, \omega, z^{0}\right)_{\tau}^{2}-\frac{\left(\left.\frac{\mathrm{d}}{\mathrm{d} t} D g_{i}(\cdot, \bar{y}) \bar{\Gamma}_{N}^{\prime}\left(v, \omega, z^{0}\right)\right|_{\tau}\right)^{2}}{\left.\frac{\mathrm{d}^{2}}{\mathrm{~d} t^{2}} g_{i}(\cdot, \bar{y})\right|_{\tau}}
\end{aligned}
$$

for any $\left(v, \omega, z^{0}\right) \in \mathcal{V}_{2} \times L^{2}\left(0, T ; R^{N}\right) \times \mathbb{R}^{n}$.

Proof. Combine [5, Lemma 23] with Remark 4.11 and Assumption 4.

The reduced partially relaxed problems The formulation is the same as for problems $\left(P_{N}\right)$, except that (i) we localize the mixed constraints $c$ and the state constraints $g$ on the domains given by Assumption 5. (ii) we replace the state constraints of order at least 2 around their touch points with the mappings $\Theta_{i, \tau}^{\varepsilon, N}$. Without loss of generality we assume that $\varepsilon^{\prime}$ given by Assumption 5 is smaller than $\varepsilon$ given by Lemma 4.13 ; $\delta^{\prime}$ is also given by Assumption 5 .

Let $N \in \mathbb{N}$. Recall that in Section 3.2 the partially relaxed problem was

$$
\min _{(u, \alpha, y, \theta) \in \mathcal{U} \times \mathcal{A}^{N} \times \mathcal{Y} \times \mathbb{R}} \theta \quad \text { subject to } 3.12-3.19 .
$$

We consider the following new constraints:

$$
\begin{array}{rll}
c_{i}\left(t, u_{t}, y_{t}\right) \leq \theta & \text { for a.a. } t \in \Delta_{c, i}^{\delta^{\prime}} & 1 \leq i \leq n_{c} \\
g_{i}\left(t, y_{t}\right) \leq \theta & \text { for a.a. } t \in \Delta_{g, i}^{\varepsilon^{\prime}} & 1 \leq i \leq n_{g}, \\
\Theta_{i, \tau}^{\varepsilon^{\prime}, N}\left(u, \alpha, y_{0}\right) \leq \theta & \text { for all } \tau \in \mathcal{T}_{g, i} & 1 \leq i \leq n_{g} .
\end{array}
$$

The reduced partially relaxed problem is then

$$
\min _{(u, \alpha, y, \theta) \in \mathcal{U} \times \mathcal{A}^{N} \times \mathcal{Y} \times \mathbb{R}} \theta \quad \text { subject to } \quad 3.12-3.15,3.18-\left(3.19,4.27-\left(4.29 . \quad\left(P_{N}^{R}\right)\right.\right.
$$

As before, we denote by $F\left(P_{N}^{R}\right)$ the set of feasible relaxed trajectories.

Remark 4.14. 1. We have $(\bar{u}, \bar{\alpha}, \bar{y}, \bar{\theta}) \in F\left(P_{N}^{R}\right)$ and, in a neighborhood of $(\bar{u}, \bar{\alpha}, \bar{y}, \bar{\theta}),(u, \alpha, y, \theta) \in$ $F\left(P_{N}^{R}\right)$ iff $(u, \alpha, y, \theta) \in F\left(P_{N}\right)$. In particular, $(\bar{u}, \bar{\alpha}, \bar{y}, \bar{\theta})$ is a weak minimum for problem $P_{N}^{R}$ iff it is a weak minimum for problem $P_{N}$.

2. Problem $\left.\sqrt{P_{N}^{R}}\right)$ is qualified at $(\bar{u}, \bar{\alpha}, \bar{y}, \bar{\theta})$ iff problem $\sqrt{P_{N}}$ is qualified at $(\bar{u}, \bar{\alpha}, \bar{y}, \bar{\theta})$ (see Remark $3.6 \cdot 2)$. 
Optimality conditions Again, problem $\left(P_{N}^{R}\right)$ can be seen as an optimization problem over $\left(u, \alpha, y^{0}, \theta\right)$, via the mapping $\Gamma_{N}$. We denote its Lagrangian by $L_{N}^{R}$, its set of Lagrange multipliers at $\left(\bar{u}, \bar{\alpha}, \bar{y}_{0}, \bar{\theta}\right)$ by $\Lambda\left(P_{N}^{R}\right)$, and its set of quasi radial critical directions in $L^{2}$ by $C_{2}^{Q R}\left(P_{N}^{R}\right)$, as defined in Appendix A.1.

Remark 4.15. By Lemma 4.13 , we can identify $\Lambda\left(P_{N}^{R}\right)$ and $\Lambda\left(P_{N}\right)$ by identifying the scalar components of a multiplier associated to the constraints 4.29 ) and Dirac measures. See also [5, Lemma 26] or [3, Lemma 3.4].

We have the following second-order necessary conditions:

Lemma 4.16. Let problem $P_{N}^{R}$ be qualified at $(\bar{u}, \bar{\alpha}, \bar{y}, \bar{\theta})$. Then for any $\left(v, \omega, z^{0}, \vartheta\right) \in \operatorname{cl}\left(C_{2}^{Q R}\left(P_{N}^{R}\right)\right)$, there exists $(\lambda, \gamma) \in \Lambda\left(P_{N}^{R}\right)$ such that

$$
D^{2} L_{N}^{R}[\lambda, \gamma]\left(v, \omega, z^{0}, \vartheta\right)^{2} \geq 0 .
$$

Here, cl denotes the $L^{2}$ closure.

Proof. We apply Theorem A.5 to $\left(\bar{u}, \bar{\alpha}, \bar{y}_{0}, \bar{\theta}\right)$, locally optimal solution of $\left(P_{N}^{R}\right)$ by Theorem 3.7 and Remark 4.14. The various mappings have the required regularity by Assumption 3 and Lemma 4.13 Robinson's contraint qualification and the inward condition for the mixed constraints hold as in the proof of Lemma 3.9 . The conclusion follows.

\subsubsection{Proof of the theorem}

Let $(\bar{v}, \bar{z}) \in C_{2}^{S}(\bar{u}, \bar{y})$. By Lemma 3.5 and since $\lambda \mapsto \Omega[\lambda](\bar{v}, \bar{z})$ is linear continuous, it is enough to show that for all $N$, there exists $\lambda^{N} \in \Lambda^{N}(\bar{u}, \bar{y})$ such that

$$
\Omega\left[\lambda^{N}\right](\bar{v}, \bar{z}) \geq 0 .
$$

Let $(\bar{\omega}, \bar{\vartheta}):=(0,0) \in \mathcal{A}^{N} \times \mathbb{R}$. The link with the reduced partially relaxed problems $\left(P_{N}^{R}\right)$ is as follows:

Lemma 4.17. Let $(\lambda, \gamma) \in \Lambda\left(P_{N}^{R}\right)$. Then $\lambda \in \Lambda^{N}(\bar{u}, \bar{y})$ and

$$
D^{2} L_{N}^{R}[\lambda, \gamma]\left(\bar{v}, \bar{\omega}, \bar{z}_{0}, \bar{\vartheta}\right)^{2}=\Omega[\lambda](\bar{v}, \bar{z}) .
$$

Proof. The first part of the result is known by Lemma 3.10 and Remark 4.15 For the second part, we write $L_{N}^{R}$ using $H^{a}$ and $H$, as in the expression $(3.28)$ of $L_{N}$, and we compute its second derivative. The result follows by Lemma 4.13 and Remark 4.12. See also [5, Lemma 26] or [3, Lemma 3.5].

We also need the following density result, that will be proved in Section 4.2 .3

Lemma 4.18. The direction $\left(\bar{v}, \bar{\omega}, \bar{z}_{0}, \bar{\vartheta}\right)$ belongs to $\operatorname{cl}\left(C_{2}^{Q R}\left(P_{N}^{R}\right)\right)$, the closure of the set of quasi radial critical directions in $L^{2}$.

We can now conclude:

Proof of Theorem 4.9 We need $\lambda^{N} \in \Lambda^{N}(\bar{u}, \bar{y})$ such that (4.31) holds for all $N$. If problem $\left(P_{N}^{R}\right)$ is qualified at $(\bar{u}, \bar{\alpha}, \bar{y}, \bar{\theta})$, then we get $\lambda^{N}$ as needed by Lemmas 4.164 .17 and 4.18 . If problem $\left(\overline{P_{N}^{R}}\right)$ is not qualified at $(\bar{u}, \bar{\alpha}, \bar{y}, \bar{\theta})$, then we get $\lambda$ such that $-\lambda, \lambda \in \Lambda^{N}(\bar{u}, \bar{y})$ by Remark 4.142 and Lemma 3.11. Since $\lambda \mapsto \Omega[\lambda](\bar{v}, \bar{z})$ is linear, 4.31 holds for $\lambda^{N}= \pm \lambda$. 


\subsubsection{A density result}

In this section we prove Lemma 4.18. Recall that $\delta^{\prime}$ is given by Assumption 5. We define the strict radial critical cone in $L^{2}$

$$
C_{2}^{R}(\bar{u}, \bar{y}):=\left\{\begin{array}{lll}
(v, z) \in C_{2}(\bar{u}, \bar{y}): & \\
\exists \delta>0: D c_{i}\left(t, \bar{u}_{t}, \bar{y}_{t}\right)\left(v_{t}, z_{t}\right)=0 & t \in \Delta_{c, i}^{\delta} \quad 1 \leq i \leq n_{c} \\
\exists M>0:\left|D c_{i}\left(t, \bar{u}_{t}, \bar{y}_{t}\right)\left(v_{t}, z_{t}\right)\right| \leq M & t \in \Delta_{c, i}^{\delta^{\prime}} \quad 1 \leq i \leq n_{c} \\
\exists \varepsilon>0: D g_{i}\left(t, \bar{y}_{t}\right) z_{t}=0 & t \in \Delta_{g, i}^{\varepsilon} \quad 1 \leq i \leq n_{g}
\end{array}\right\} .
$$

Proposition 4.19. The strict radial critical cone $C_{2}^{R}(\bar{u}, \bar{y})$ is a dense subset of the strict critical cone $C_{2}^{S}(\bar{u}, \bar{y})$.

Proof. Touch points for $g_{i}$ are included in $\Delta_{g, i}^{\varepsilon}, \varepsilon \geq 0$, iff $g_{i}$ is of order 1 .

(a) Let $W^{(q), 2}(0, T):=\prod_{i=1}^{n_{g}} W^{q_{i}, 2}(0, T)$. We claim that the subspace

$$
\left\{\begin{array}{l}
(\phi, \psi) \in L^{\infty}\left(0, T ; \mathbb{R}^{n_{c}}\right) \times W^{(q), 2}(0, T): \\
\exists \delta>0: \phi_{i, t}=0 \quad t \in \Delta_{c, i}^{\delta} \quad 1 \leq i \leq n_{c} \\
\exists \varepsilon>0: \psi_{i, t}=0 \quad t \in \Delta_{g, i}^{\varepsilon} \quad 1 \leq i \leq n_{g}
\end{array}\right\}
$$

is a dense subset of

$$
\left\{\begin{array}{c}
(\phi, \psi) \in L^{2}\left(0, T ; \mathbb{R}^{n_{c}}\right) \times W^{(q), 2}(0, T): \\
\phi_{i, t}=0 \quad t \in \Delta_{c, i}^{0} \quad 1 \leq i \leq n_{c} \\
\psi_{i, t}=0 \quad t \in \Delta_{g, i}^{0} \quad 1 \leq i \leq n_{g}
\end{array}\right\}
$$

Indeed, for $\phi_{i} \in L^{2}(0, T)$, we consider the sequence

$$
\phi_{i, t}^{k}:= \begin{cases}0 & \text { if } t \in \Delta_{c, i}^{1 / k} \\ \min \left\{k,\left|\phi_{i, t}\right|\right\} \frac{\phi_{i, t}}{\left|\phi_{i, t}\right|} & \text { otherwise }\end{cases}
$$

For $\psi_{i} \in W^{q_{i}, 2}(0, T)$, we use the fact that there is no isolated point in $\Delta_{g, i}^{0}$ if $q_{i} \geq 2$, and approximation results in $W^{q_{i}, 2}(0, T)$, e.g. [3, Appendix A.3]. Our claim follows.

(b) By Assumption 5 and the open mapping theorem, there exists $C>0$ such that for all $(\phi, \psi) \in L^{2}\left(0, T ; \mathbb{R}^{n_{c}}\right) \times W^{(q), 2}(0, T)$, there exists $(v, z) \in \mathcal{V}_{2} \times \mathcal{Z}_{2}$ such that

$$
\begin{aligned}
& z=z\left[v, z_{0}\right], \quad\|v\|_{2}+\left|z_{0}\right| \leq C\left(\|\phi\|_{2}+\|\psi\|_{(q), 2}\right) \\
& D c_{i}\left(t, \bar{u}_{t}, \bar{y}_{t}\right)\left(v_{t}, z_{t}\right)=\phi_{i, t} \quad t \in \Delta_{c, i}^{\delta^{\prime}} \quad 1 \leq i \leq n_{c} \\
& D g_{i}\left(t, \bar{y}_{t}\right) z_{t}=\psi_{i, t} \\
& t \in \Delta_{g, i}^{\varepsilon^{\prime}} \quad 1 \leq i \leq n_{g} .
\end{aligned}
$$

It follows that the subspace

$$
\left\{\begin{array}{lll}
(v, z) \in \mathcal{V}_{2} \times \mathcal{Z}_{2}: z=z\left[v, z_{0}\right] & \\
\exists \delta>0 \quad: D c_{i}\left(t, \bar{u}_{t}, \bar{y}_{t}\right)\left(v_{t}, z_{t}\right)=0 & t \in \Delta_{c, i}^{\delta} & 1 \leq i \leq n_{c} \\
\exists M>0:\left|D c_{i}\left(t, \bar{u}_{t}, \bar{y}_{t}\right)\left(v_{t}, z_{t}\right)\right| \leq M & t \in \Delta_{c, i}^{\delta^{\prime}} & 1 \leq i \leq n_{c} \\
\exists \varepsilon>0: D g_{i}\left(t, \bar{y}_{t}\right) z_{t}=0 & t \in \Delta_{g, i}^{\varepsilon} & 1 \leq i \leq n_{g}
\end{array}\right\}
$$

is a dense subset of

RR n 8306




Observe now that $C_{2}^{R}(\bar{u}, \bar{v})$ and $C_{2}^{S}(\bar{u}, \bar{v})$ are defined by 4.33) and 4.17) respectively as the same polyhedral cone in the previous two vector spaces. See also Remark 4.8 2. Then by [14, Lemma 1], the conclusion of Proposition 4.19 follows.

The definition of the set $C_{2}^{Q R}\left(P_{N}^{R}\right)$ of quasi radial critical directions in $L^{2}$ is given in Appendix A.1. Recall that $(\bar{\omega}, \bar{\vartheta}):=(0,0) \in \mathcal{A}^{N} \times \mathbb{R}$.

Lemma 4.20. Let $(v, z) \in C_{2}^{R}(\bar{u}, \bar{y})$. Then $\left(v, \bar{\omega}, z_{0}, \bar{\vartheta}\right) \in C_{2}^{Q R}\left(P_{N}^{R}\right)$.

Proof. The direction $\left(v, \bar{\omega}, z_{0}, \bar{\vartheta}\right)$ is radial [8, Definition 3.52] for the finite dimensional constraints, which are polyhedral, as well as for the constraints on $\alpha$. Let $\delta$ and $M>0$ be given by definition of $C_{2}^{R}(\bar{u}, \bar{y})$. Then for any $\sigma>0$

$$
c_{i}\left(t, \bar{u}_{t}, \bar{y}_{t}\right)+\sigma D c_{i}\left(t, \bar{u}_{t}, \bar{y}_{t}\right)\left(v_{t}, z_{t}\right) \leq \begin{cases}0 & \text { for a.a. } t \in \Delta_{c, i}^{\delta} \\ -\delta+\sigma M & \text { for a.a. } t \in \Delta_{c, i}^{\delta^{\prime}} \backslash \Delta_{c, i}^{\delta}\end{cases}
$$

i.e. $\left(v, \bar{\omega}, z_{0}, \bar{\vartheta}\right)$ is radial for the constraint 4.27). The same argument holds for constraint 4.28 since there exists $\delta_{0}>0$ such that $g_{i}\left(t, \bar{y}_{t}\right) \leq-\delta_{0}$ for all $t \in \Delta_{g, i}^{\varepsilon^{\prime}} \backslash \Delta_{g, i}^{\varepsilon}$. Then $\left(v, \bar{\omega}, z_{0}, \vartheta\right)$ is radial, and a fortiori quasi radial.

Remark 4.21. To finish this section, let us mention a flaw in the proof of the density result 6 , Lemma 6.4 (ii)]. There is no reason that $v^{n}$ belongs to $L^{\infty}$, and not only to $L^{2}$, since $\left(v^{n}-v\right)$ is obtained as a preimage of $\left(w^{n}-w, \omega^{n}-\omega\right)$. The lemma is actually true but its proof requires some effort, see [3. Lemma 4.5] for the case without mixed constraints. The difficulty is avoided here because we do not have to show the density of a $L^{\infty}$ cone, thanks to our abstract second-order necessary conditions, Theorem A.5, that are derived directly in $L^{2}$.

\section{A Appendix}

\section{A.1 Abstract optimization results}

In this section, we recall necessary conditions satified by a weak minimum of a general optimal control problem. These conditions have been used in this paper to prove our necessary conditions in Pontryagin form, namely Theorems 3.1 and 4.9 , via the partial relaxation, i.e. Lemmas 3.9 and 4.16 .

We actually state and prove first- and second-order necessary conditions for a more abstract optimization problem. It has to be noted that our second-order conditions, Theorem A.5 are obtained directly on a large set of directions in $L^{2}$, thanks to metric regularity result, Lemma A.7 and a tricky truncation, Lemma A.8. To our knowledge, this is new.

\section{A.1.1 Setting}

Let $K$ be a nonempty closed convex subset of a Banach space $X$ and $\Delta_{1}, \ldots, \Delta_{M}$ be measurable sets of $[0, T]$. For $s \in[1, \infty]$, let

$$
\begin{array}{ll}
\mathcal{U}_{s}:=L^{s}\left(0, T ; \mathbb{R}^{\bar{m}}\right), & \mathcal{Y}_{s}:=W^{1, s}\left(0, T ; \mathbb{R}^{\bar{n}}\right) \\
X_{s}:=X \times \prod_{i=1}^{M} L^{s}\left(\Delta_{i}\right), & K_{s}:=K \times \prod_{i=1}^{M} L^{s}\left(\Delta_{i} ; \mathbb{R}_{-}\right) .
\end{array}
$$


We consider

$$
\begin{aligned}
\Gamma: \mathcal{U}_{\infty} \times \mathbb{R}^{\bar{n}} \rightarrow \mathcal{Y}_{\infty}, \quad J: \mathcal{U}_{\infty} \times \mathbb{R}^{\bar{n}} \rightarrow \mathbb{R}, \\
G_{1}: \mathcal{U}_{\infty} \times \mathbb{R}^{\bar{n}} \rightarrow X, \quad G_{2}^{i}: \mathcal{U}_{\infty} \times \mathcal{Y}_{\infty} \rightarrow L^{\infty}\left(\Delta_{i}\right),
\end{aligned}
$$

the last mappings being defined for $i=1, \ldots, M$ by

$$
G_{2}^{i}(u, y)_{t}:=m_{i}\left(t, u_{t}, y_{t}\right)
$$

for a.a. $t \in \Delta_{i}$, where $m_{i}:[0, T] \times \mathbb{R}^{\bar{m}} \times \mathbb{R}^{\bar{n}} \rightarrow \mathbb{R}$. Let

$$
G: \mathcal{U}_{\infty} \times \mathbb{R}^{\bar{n}} \rightarrow X_{\infty}, \quad G\left(u, y^{0}\right):=\left(G_{1}\left(u, y^{0}\right), G_{2}\left(u, \Gamma\left(u, y^{0}\right)\right) .\right.
$$

The optimization problem we consider is the following:

$$
\min _{\left(u, y^{0}\right) \in \mathcal{U}_{\infty} \times \mathbb{R}^{\bar{n}}} J\left(u, y^{0}\right) ; G\left(u, y^{0}\right) \in K_{\infty} .
$$

Remark A.1. Optimal control problems fit into this framework as follows: given a uniformly quasi- $C^{1}$ mapping $F: \mathbb{R} \times \mathbb{R}^{\bar{m}} \times \mathbb{R}^{\bar{n}} \rightarrow \mathbb{R}^{\bar{n}}$ and the state equation

$$
\dot{y}_{t}=F\left(t, u_{t}, y_{t}\right) \quad \text { for a.a. } t \in(0, T) \text {, }
$$

we define $\Gamma\left(u, y^{0}\right)$ as the unique $y \in \mathcal{Y}_{\infty}$ such that A.7 holds and $y_{0}=y^{0}$, for any $\left(u, y^{0}\right) \in$ $\mathcal{U}_{\infty} \times \mathbb{R}^{\bar{n}}$; given a cost function $\widetilde{J}: \mathcal{Y}_{\infty} \rightarrow \mathbb{R}$, we define $J:=\widetilde{J} \circ \Gamma$; given state constraints of any kind (pure, initial-final, ...) $\widetilde{G}_{1}: \mathcal{Y}_{\infty} \rightarrow X$, with the appropriate space $X$ and convex subset $K$, we define $G_{1}:=\widetilde{G}_{1} \circ \Gamma$; finally, we define $G_{2}$ in order to take into account the mixed control-state and control constraints. By definition, a weak minimum of such an optimal control problem is a locally optimal solution of the corresponding optimization problem $(A P)$.

\section{A.1.2 Assumptions}

Let $\left(\bar{u}, \bar{y}^{0}\right)$ be feasible for $A P$ and let $\bar{y}:=\Gamma\left(\bar{u}, \bar{y}^{0}\right)$. For various Banach spaces $Y$ and mappings $\mathcal{F}: \mathcal{U}_{\infty} \times \mathbb{R}^{\bar{n}} \rightarrow Y$, we will require one of the followings:

Property 1. The mapping $\mathcal{F}$ is $C^{1}$ in a neighborhood of $\left(\bar{u}, \bar{y}^{0}\right)$, with continuous extensions $D \mathcal{F}\left(u, y^{0}\right): \mathcal{U}_{1} \times \mathbb{R}^{\bar{n}} \rightarrow Y$.

Property 2. Property 1 holds, and $\mathcal{F}$ is twice Fréchet differentiable at $\left(\bar{u}, \bar{y}^{0}\right)$, with a continuous extension $D^{2} \mathcal{F}\left(\bar{u}, \bar{y}^{0}\right):\left(\mathcal{U}_{2} \times \mathbb{R}^{\bar{n}}\right)^{2} \rightarrow Y$ and the following expansion in $Y$ : for all $\left(v, z^{0}\right) \in$ $\mathcal{U}_{\infty} \times \mathbb{R}^{\bar{n}}$

$$
\begin{aligned}
\mathcal{F}\left(\bar{u}+v, \bar{y}^{0}+z^{0}\right)=\mathcal{F}\left(\bar{u}, \bar{y}^{0}\right)+D \mathcal{F}\left(\bar{u}, \bar{y}^{0}\right)\left(v, z^{0}\right)+\frac{1}{2} D^{2} \mathcal{F}\left(\bar{u}, \bar{y}^{0}\right)\left(v, z^{0}\right)^{2} & \\
& +o_{\infty}\left(\|v\|_{2}^{2}+\left|z^{0}\right|^{2}\right) .
\end{aligned}
$$

Assumption (i ). The mappings $\Gamma, J$ and $G_{1}$ satisfy Property 1 , and the functions $m_{i}$ are uniformly quasi- $C^{1}$.

Assumption (i'). The mappings $\Gamma, J$ and $G_{1}$ satisfy Property 2 and the functions $m_{i}$ are uniformly quasi- $C^{2}$.

Assumption (ii). Robinson's constraint qualification holds:

$$
0 \in \operatorname{int}_{X_{\infty}}\left\{G\left(\bar{u}, \bar{y}^{0}\right)+D G\left(\bar{u}, \bar{y}^{0}\right)\left(\mathcal{U}_{\infty} \times \mathbb{R}^{\bar{n}}\right)-K_{\infty}\right\} .
$$

$\operatorname{RR} n^{\circ} 8306$ 
Assumption (iii). The inward condition holds for $G_{2}$ : there exists $\gamma>0$ and $\hat{v} \in \mathcal{U}_{\infty}$ such that

$$
G_{2}^{i}(\bar{u}, \bar{y})+D_{u} G_{2}^{i}(\bar{u}, \bar{y}) \hat{v} \leq-\gamma
$$

on $\Delta_{i}, i=1, \ldots, M$.

Remark A.2. Let us consider the case of an optimal control problem, with $\Gamma, J$ and $G_{1}$ defined as in Remark A.1. If $F, m_{i}$ are uniformly quasi- $C^{1}$ and $\widetilde{J}, \widetilde{G}_{1}$ are $C^{1}$, then Assumption (i) holds. If $F, m_{i}$ are uniformly quasi- $C^{2}$ and $\widetilde{J}, \widetilde{G}_{1}$ are $C^{2}$, then Assumption (i') holds. See for example [5. Lemmas 19-20] or [29, Theorems 3.3-3.5].

\section{A.1.3 Necessary conditions}

We consider the Lagrangian $L[\lambda]: \mathcal{U}_{\infty} \times \mathbb{R}^{\bar{n}} \rightarrow \mathbb{R}$, defined for $\lambda \in X_{\infty}^{*}$ by

$$
L[\lambda]\left(u, y^{0}\right):=J\left(u, y^{0}\right)+\left\langle\lambda, G\left(u, y^{0}\right)\right\rangle .
$$

We define the set of Lagrange multipliers as

$$
\Lambda A P:=\left\{\lambda \in X_{1}^{*}: \lambda \in N_{K_{1}}\left(G\left(\bar{u}, \bar{y}^{0}\right)\right), D L[\lambda]\left(\bar{u}, \bar{y}^{0}\right)=0 \text { on } \mathcal{U}_{1} \times \mathbb{R}^{\bar{n}}\right\}
$$

and the set of quasi radial critical directions in $L^{2}$ as

$$
C_{2}^{Q R} A P:=\left\{\begin{array}{c}
\left(v, z^{0}\right) \in \mathcal{U}_{2} \times \mathbb{R}^{\bar{n}}: D J\left(\bar{u}, \bar{y}^{0}\right)\left(v, z^{0}\right) \leq 0 \text { and } \forall \sigma>0 \\
\operatorname{dist}_{X_{1}}\left(G\left(\bar{u}, \bar{y}^{0}\right)+\sigma D G\left(\bar{u}, \bar{y}^{0}\right)\left(v, z^{0}\right), K_{1}\right)=o\left(\sigma^{2}\right)
\end{array}\right\} .
$$

We denote by $\operatorname{cl}\left(C_{2}^{Q R}(A P)\right)$ its closure in $\mathcal{U}_{2} \times \mathbb{R}^{\bar{n}}$.

Remark A.3. If $\left(v, z^{0}\right) \in C_{2}^{Q R}(A P)$, then $D G\left(\bar{u}, \bar{y}^{0}\right)\left(v, z^{0}\right) \in T_{K_{1}}\left(G\left(\bar{u}, \bar{y}^{0}\right)\right)$. If in addition $\Lambda A P \neq \emptyset$, then $D J\left(\bar{u}, \bar{y}^{0}\right)\left(v, z^{0}\right)=0$.

We now state our first- and second-order necessary conditions, in two theorems that will be proved in the next section.

Theorem A.4. Let $\left(\bar{u}, \bar{y}^{0}\right)$ be a locally optimal solution of $A P$, and let Assumptions (i)-(iii) hold. Then $\Lambda A P$ is nonempty, convex, and weakly * compact in $X_{1}^{*}$.

Theorem A.5. Let $\left(\bar{u}, \bar{y}^{0}\right)$ be a locally optimal solution of $A P$, and let Assumptions (i')-(iii) hold. Then for any $\left(v, z^{0}\right) \in \operatorname{cl}\left(C_{2}^{Q R}(A P)\right)$, there exists $\lambda \in \Lambda(A P)$ such that

$$
D^{2} L[\lambda]\left(\bar{u}, \bar{y}^{0}\right)\left(v, z^{0}\right)^{2} \geq 0
$$

\section{A.1.4 Proofs}

Proof of Theorem A.4 Robinson's constraint qualification (A.9] and [30, Theorem 4.1] or [8, Theorem 3.9] give the result in $X_{\infty}^{*}$. We derive it in $X_{1}^{*}$ with the inward condition A.10, see e.g. [7, Theorem 3.1].

Proof of Theorem A.5. (a) Assume first that $\left(v, z^{0}\right) \in C_{2}^{Q R} A P$. We consider the following conic linear problem, [8, Section 2.5.6]:

$$
\left\{\begin{array}{c}
\min _{\left(w, \xi^{0}\right) \in \mathcal{U}_{1} \times \mathbb{R}^{\bar{n}}} D J\left(\bar{u}, \bar{y}^{0}\right)\left(w, \xi^{0}\right)+D^{2} J\left(\bar{u}, \bar{y}^{0}\right)\left(v, z^{0}\right)^{2} \\
D G\left(\bar{u}, \bar{y}^{0}\right)\left(w, \xi^{0}\right)+D^{2} G\left(\bar{u}, \bar{y}^{0}\right)\left(v, z^{0}\right)^{2} \in T_{K_{1}}\left(G\left(\bar{u}, \bar{y}^{0}\right)\right) .
\end{array}\right.
$$


Robinson's constraint qualification $A .9$ for problem $A P$ implies that the constraints of $Q_{\left(v, z^{0}\right)}$ are regular in the sense of [8, Theorem 2.187]. Then by the same theorem, there is no duality gap between $Q_{\left(v, z^{0}\right)}$ and its dual, which is the following optimization problem:

$$
\max _{\lambda \in \Lambda(A P)} D^{2} L[\lambda]\left(\bar{u}, \bar{y}^{0}\right)\left(v, z^{0}\right)^{2} .
$$

Observe indeed that the Lagrangian of $Q_{\left(v, z^{0}\right)}$ is

$$
\mathcal{L}[\lambda]\left(w, \xi^{0}\right)=D L[\lambda]\left(\bar{u}, \bar{y}^{0}\right)\left(w, \xi^{0}\right)+D^{2} L[\lambda]\left(\bar{u}, \bar{y}^{0}\right)\left(v, z^{0}\right)^{2}, \quad \lambda \in X_{1}^{*} .
$$

The conclusion of the theorem follows when $\left(v, z^{0}\right) \in C_{2}^{Q R}(A P)$ by the following key lemma, that will be proved below.

Lemma A.6. The value of $\sqrt{Q_{\left(v, z^{0}\right)}}$ is nonnegative.

(b) Assume now that $\left(v, z^{0}\right) \in \operatorname{cl}\left(C_{2}^{Q R}(A P)\right)$. Let $\left(v^{k}, z^{0, k}\right) \in C_{2}^{Q R}\left(A P\right.$ converge to $\left(v, z^{0}\right)$ in $\mathcal{U}_{2} \times \mathbb{R}^{\bar{n}}$. By step (b), there exists $\lambda^{k} \in \Lambda$ be such that

$$
\begin{aligned}
D^{2} J\left(\bar{u}, \bar{y}^{0}\right)\left(v^{k}, z^{0, k}\right)^{2}+\left\langle\lambda^{k}, D^{2} G\left(\bar{u}, \bar{y}^{0}\right)\left(v^{k}, z^{0, k}\right)^{2}\right\rangle & \\
& =D^{2} L\left[\lambda^{k}\right]\left(\bar{u}, \bar{y}^{0}\right)\left(v^{k}, z^{0, k}\right)^{2} \geq 0 .
\end{aligned}
$$

By Theorem A.4 there exists $\lambda \in \Lambda$ such that, up to a subsequence, $\lambda^{k} \rightarrow \lambda$ for the weak $*$ topology in $X_{1}^{*}$. By Assumption (i'), $D^{2} J\left(\bar{u}, \bar{y}^{0}\right): \mathcal{U}_{2} \times \mathbb{R}^{\bar{n}} \rightarrow \mathbb{R}$ and $D^{2} G\left(\bar{u}, \bar{y}^{0}\right): \mathcal{U}_{2} \times \mathbb{R}^{\bar{n}} \rightarrow X_{1}$ are continuous. The conclusion follows.

Proof of Lemma A.6. First we prove a metric regularity result, which relies on Assumption (iii). For any $(u, y) \in \mathcal{U}_{\infty} \times \mathcal{Y}_{\infty}$, we define $G_{2}^{+}(u, y) \in L^{\infty}(0, T)$ by

$$
G_{2}^{+}(u, y)_{t}:=\max _{1 \leq i \leq M}\left(G_{2}^{i}(u, y)_{t}\right)_{+}
$$

for a.a. $t \in(0, T)$, where

$$
\left(G_{2}^{i}(u, y)_{t}\right)_{+}:= \begin{cases}\max \left\{0, G_{2}^{i}(u, y)_{t}\right\} & \text { if } t \in \Delta_{i} \\ 0 & \text { if } t \notin \Delta_{i}\end{cases}
$$

Lemma A.7. There exists $c>0$ such that, for any $(u, y) \in \mathcal{U}_{\infty} \times \mathcal{Y}_{\infty}$ with $y=\Gamma\left(u, y^{0}\right)$ in a neighborhood of $(\bar{u}, \bar{y})$, there exists $(\hat{u}, \hat{y}) \in \mathcal{U}_{\infty} \times \mathcal{Y}_{\infty}$ with $\hat{y}=\Gamma\left(\hat{u}, y^{0}\right)$ such that

$$
\begin{aligned}
\|\hat{u}-u\|_{\infty} & \leq c\left\|G_{2}^{+}(u, y)\right\|_{\infty}, \\
\|\hat{u}-u\|_{1} & \leq c\left\|G_{2}^{+}(u, y)\right\|_{1}, \\
\left\|G_{2}^{+}(\hat{u}, \hat{y})\right\|_{\infty} & \leq c\left\|G_{2}^{+}(u, y)\right\|_{1} .
\end{aligned}
$$

Proof. Let $\beta \in(0,1)$ to be fixed later. Since $\left(\bar{u}, \bar{y}^{0}\right)$ is feasible, $G_{2}^{+}(\bar{u}, \bar{y})=0$, and there exists $\alpha \in(0, \beta)$ such that

$$
\|u-\bar{u}\|_{\infty}+\|y-\bar{y}\|_{\infty} \leq \alpha \Rightarrow\left\|G_{2}^{+}(u, y)\right\|_{\infty} \leq \beta
$$

Let $(u, y)$ be such that $\|u-\bar{u}\|_{\infty}+\|y-\bar{y}\|_{\infty} \leq \alpha$. We define $\varepsilon \in L^{\infty}(0, T)$ by

$$
\varepsilon_{t}:=\frac{1}{\beta} G_{2}^{+}(u, y)_{t},
$$

$\mathrm{RR} \mathrm{n}^{\circ} 8306$ 
so that $\varepsilon_{t} \in[0,1]$ for a.a. $t \in(0, T)$, and

$$
\hat{u}:=u+\varepsilon \hat{v}
$$

where $\hat{v}$ is given by the inward condition A.10. Once $\beta$ is fixed, it is clear that A.20 and A.21 hold. Let $\hat{y}=\Gamma\left(\hat{u}, y^{0}\right)$.

$$
\begin{aligned}
G_{2}^{i}(\hat{u}, \hat{y})=G_{2}^{i} & (u, y)+D G_{2}^{i}(\bar{u}, \bar{y})(\hat{u}-u, \hat{y}-y) \\
& +\int_{0}^{1}\left(D G_{2}^{i}(u+\theta(\hat{u}-u), y+\theta(\hat{y}-y))-D G_{2}^{i}(\bar{u}, \bar{y})\right)(\hat{u}-u, \hat{y}-y) \mathrm{d} \theta
\end{aligned}
$$

a.e. on $\Delta_{i}$. Since $\Gamma$ satisfies Property $1,\|\hat{y}-y\|_{\infty}=O\left(\|\hat{u}-u\|_{1}\right)$, and then

$$
\begin{aligned}
\left|\hat{u}_{t}-u_{t}\right| & =O\left(\varepsilon_{t}\right), & \left|u_{t}-\bar{u}_{t}\right| & =O(\alpha)=O(\beta), \\
\left|\hat{y}_{t}-y_{t}\right| & =O\left(\|\varepsilon\|_{1}\right), & \left|y_{t}-\bar{y}_{t}\right| & =O(\alpha)=O(\beta) .
\end{aligned}
$$

Since $m_{i}$ is uniformly quasi- $C^{2}, G_{2}^{i}$ and $D G_{2}^{i}$ are Lipschitz in a neighborhood of $(\bar{u}, \bar{y})$. Then

$$
\begin{aligned}
G_{2}^{i}(\hat{u}, \hat{y})= & G_{2}^{i}(u, y)+\varepsilon D_{u} G_{2}^{i}(\bar{u}, \bar{y}) \hat{v}+O\left(\|\varepsilon\|_{1}+\varepsilon\left(\varepsilon+\|\varepsilon\|_{1}+\beta\right)\right) \\
= & (1-\varepsilon) G_{2}^{i}(u, y)+\varepsilon\left(G_{2}^{i}(u, y)-G_{2}^{i}(\bar{u}, \bar{y})\right) \\
& +\varepsilon\left(G_{2}^{i}(\bar{u}, \bar{y})+D_{u} G_{2}^{i}(\bar{u}, \bar{y}) \hat{v}\right)+O\left(\|\varepsilon\|_{1}+\varepsilon\left(\varepsilon+\|\varepsilon\|_{1}+\beta\right)\right) .
\end{aligned}
$$

Observe now that

$$
\begin{gathered}
(1-\varepsilon) G_{2}^{i}(u, y) \leq G_{2}^{+}\left(u, y^{0}\right)=\varepsilon \beta, \\
\varepsilon\left(G_{2}^{i}(u, y)-G_{2}^{i}(\bar{u}, \bar{y})\right)=O(\alpha \varepsilon)=O(\varepsilon \beta), \\
\varepsilon\left(G_{2}^{i}(\bar{u}, \bar{y})+D_{u} G_{2}^{i}(\bar{u}, \bar{y}) \hat{v}\right) \leq-\varepsilon \gamma .
\end{gathered}
$$

Then there exists $C>0$, independent of $u$ and $u^{\prime}$, such that

$$
G_{2}^{i}(\hat{u}, \hat{y}) \leq C\|\varepsilon\|_{1}+\varepsilon\left[C\left(\varepsilon+\|\varepsilon\|_{1}+\beta\right)-\gamma\right]
$$

on $\Delta_{i}, i=1, \ldots, M$. We fix $\beta \in(0,1)$ such that $C \beta \leq \gamma / 2$ and $\alpha \in(0, \beta)$ such that $C\left(\varepsilon+\|\varepsilon\|_{1}\right) \leq$ $\gamma / 2$. The result follows.

To prove Lemma A.6. we also need the following:

Lemma A.8. Let $v \in \mathcal{U}_{2}$ and $w \in \mathcal{U}_{1}$. Let $v^{k}:=\mathbf{1}_{\{|v| \leq k\}} v, w^{k}:=\mathbf{1}_{\{|w| \leq k\}} w$, and $\sigma_{k}:=\frac{\left\|v^{k}-v\right\|_{2}}{k}$. Then $v^{k}, w^{k} \in \mathcal{U}_{\infty}, \sigma_{k} \rightarrow 0$, and

$$
\begin{aligned}
\left\|\sigma_{k} v^{k}\right\|_{\infty} & =o(1), \quad\left\|\sigma_{k}^{2} w^{k}\right\|_{\infty}=o(1), \\
\left\|v^{k}-v\right\|_{2} & =o(1), \quad\left\|w^{k}-w\right\|_{1}=o(1), \\
\left\|v^{k}-v\right\|_{1} & =o\left(\sigma_{k}\right) .
\end{aligned}
$$

Proof. We first get A.36 by Lebesgue's dominated convergence theorem. Then $\sigma_{k}=o\left(\frac{1}{k}\right)$, and A.35 follows. Observe that $\left|v^{k}-v\right|^{2} \geq k\left|v^{k}-v\right|$, which implies $\left\|v^{k}-v\right\|_{1}=O\left(\frac{1}{k}\left\|v^{k}-v\right\|_{2}^{2}\right)$. A.37 follows by definition of $\sigma_{k}$ and by A.36. 
Let us now go back to the proof of Lemma A.6. let $\left(w, \xi^{0}\right)$ be feasible for problem $\overline{\left.Q_{\left(v, z^{0}\right)}\right)}$. We apply Lemma A.8 to $v \in \mathcal{U}_{2}, w \in \mathcal{U}_{1}$, and we consider

$$
\begin{aligned}
u^{k} & :=\bar{u}+\sigma_{k} v^{k}+\frac{1}{2} \sigma_{k}^{2} w^{k} \in \mathcal{U}_{\infty}, \\
y^{0, k} & :=\bar{y}^{0}+\sigma_{k} z^{0}+\frac{1}{2} \sigma_{k}^{2} \xi^{0} \in \mathbb{R}^{\bar{n}}, \\
y^{k} & :=\Gamma\left(u^{k}, y^{0, k}\right) \in \mathcal{Y}_{\infty} .
\end{aligned}
$$

We have in particular

$$
\left\|u^{k}-\bar{u}\right\|_{\infty}=o(1), \quad\left\|u^{k}-\bar{u}\right\|_{2}=O\left(\sigma_{k}\right) .
$$

By analogy with linearized trajectories, we denote

$$
z\left[\tilde{v}, \tilde{z}^{0}\right]:=D \Gamma\left(\bar{u}, \bar{y}^{0}\right)\left(\tilde{v}, \tilde{z}^{0}\right), \quad z^{2}\left[\tilde{v}, \tilde{z}_{0}\right]:=D^{2} \Gamma\left(\bar{u}, \bar{y}^{0}\right)\left(\tilde{v}, \tilde{z}^{0}\right)^{2}
$$

for any $\left(\tilde{v}, \tilde{z}^{0}\right) \in \mathcal{U}_{\infty} \times \mathbb{R}^{\bar{n}}$. Since $\Gamma$ satisfies Property 2, we have in $\mathcal{Y}_{\infty}$

$$
y^{k}=\bar{y}+\sigma_{k} z\left[v^{k}, z^{0}\right]+\frac{1}{2} \sigma_{k}^{2}\left(z\left[w^{k}, \xi^{0}\right]+z^{2}\left[v^{k}, z^{0}\right]\right)+o\left(\sigma_{k}^{2}\right),
$$

and in particular, $\left\|y^{k}-\bar{y}\right\|_{\infty}=O\left(\sigma_{k}\right)$. Then $\left(u^{k}, y^{k}\right) \rightarrow(\bar{u}, \bar{y})$ in $\mathcal{U}_{\infty} \times \mathcal{Y}_{\infty}$ and

$$
\left\|G_{2}^{+}\left(u^{k}, y^{k}\right)\right\|_{\infty}=o(1) .
$$

More precisely, since $m_{i}$ is uniformly quasi- $C^{2}$, we have

$$
\begin{aligned}
G_{2}^{i}\left(u^{k}, y^{k}\right)=G_{2}^{i}(\bar{u}, \bar{y})+ & D G_{2}^{i}(\bar{u}, \bar{y})\left(u^{k}-\bar{u}, y^{k}-\bar{y}\right) \\
& +\frac{1}{2} D^{2} G_{2}^{i}(\bar{u}, \bar{y})\left(u^{k}-\bar{u}, y^{k}-\bar{y}\right)^{2}+o\left(\left|u^{k}-\bar{u}\right|^{2}+\left|y^{k}-\bar{y}\right|^{2}\right)
\end{aligned}
$$

a.e. on $\Delta_{i}$, where $o(\cdot)$ is uniform w.r.t. $t$. We write

$$
G_{2}^{i}\left(u^{k}, y^{k}\right)_{t}=\frac{1}{2} T_{t}^{i, k}+\frac{1}{2} Q_{t}^{i, k}+R_{t}^{i, k}
$$

where, omitting the time argument $t$,

$$
\begin{aligned}
T^{i, k}:= & G_{2}^{i}(\bar{u}, \bar{y})+2 \sigma_{k} D G_{2}^{i}(\bar{u}, \bar{y})\left(v^{k}, z\left[v^{k}, z^{0}\right]\right) \\
Q^{i, k}:= & G_{2}^{i}(\bar{u}, \bar{y})+\sigma_{k}^{2}\left(D G_{2}^{i}(\bar{u}, \bar{y})\left(w^{k}, z\left[w^{k}, \xi^{0}\right]\right)\right. \\
& \left.\quad+D^{2} G_{2}^{i}(\bar{u}, \bar{y})\left(v^{k}, z\left[v^{k}, z^{0}\right]\right)^{2}+D_{y} G_{2}^{i}(\bar{u}, \bar{y}) z^{2}\left[v^{k}, z^{0}\right]\right) \\
R^{i, k}= & \frac{1}{2} \sigma_{k}^{3} D^{2} G_{2}^{i}(\bar{u}, \bar{y})\left[\left(v^{k}, z\left[v^{k}, z^{0}\right]\right),\left(w^{k}, z\left[w^{k}, \xi^{0}\right]+z^{2}\left[v^{k}, z^{0}\right]+o(1)\right)\right] \\
& +\frac{1}{4} \sigma_{k}^{4} D^{2} G_{2}^{i}(\bar{u}, \bar{y})\left(w^{k}, z\left[w^{k}, \xi^{0}\right]+z^{2}\left[v^{k}, z^{0}\right]+o(1)\right)^{2} \\
& \quad+o\left(\left|u^{k}-\bar{u}\right|^{2}+\left|y^{k}-\bar{y}\right|^{2}\right)
\end{aligned}
$$

We claim that $\left\|R^{i, k}\right\|_{1}=o\left(\sigma_{k}^{2}\right)$. Indeed, $z\left[v^{k}, z^{0}\right], z\left[w^{k}, \xi^{0}\right]$ and $z^{2}\left[v^{k}, z^{0}\right]$ are bounded in $\mathcal{Y}_{\infty}$; the crucial terms are then the following:

$$
\begin{aligned}
\left\|\sigma_{k}^{3} D_{u u}^{2} G_{2}^{i}(\bar{u}, \bar{y})\left(v^{k}, w^{k}\right)\right\|_{1} & =O\left(\left\|\sigma_{k} v^{k}\right\|_{\infty} \cdot\left\|\sigma_{k}^{2} w^{k}\right\|_{1}\right)=o\left(\sigma_{k}^{2}\right) \\
\left\|\sigma_{k}^{4} D_{u u}^{2} G_{2}^{i}(\bar{u}, \bar{y})\left(w^{k}, w^{k}\right)\right\|_{1} & =O\left(\left\|\sigma_{k}^{2} w^{k}\right\|_{\infty} \cdot\left\|\sigma_{k}^{2} w^{k}\right\|_{1}\right)=o\left(\sigma_{k}^{2}\right) \\
\left\|o\left(\left|u^{k}-\bar{u}\right|^{2}+\left|y^{k}-\bar{y}\right|^{2}\right)\right\|_{1} & =o\left(\left\|u^{k}-\bar{u}\right\|_{2}^{2}+\left\|y^{k}-\bar{y}\right\|_{2}^{2}\right)=o\left(\sigma_{k}^{2}\right)
\end{aligned}
$$

$\mathrm{RR} n^{\circ} 8306$ 
by A.35, A.36) and A.41, A.43). Recall that $\left(v, z^{0}\right) \in C_{2}^{Q R}(A P)$. Then by A.37) and Property 1 , satisfied by $\Gamma$, we have

$$
\operatorname{dist}_{L^{1}}\left(T^{i, k}, L^{1}\left(\Delta_{i} ; \mathbb{R}_{-}\right)\right)=o\left(\sigma_{k}^{2}\right) .
$$

Similarly, since $\left(w, \xi^{0}\right)$ is feasible for $Q_{\left(v, z^{0}\right)}$ and $\Gamma$ satisfies Property 2,

$$
\operatorname{dist}_{L^{1}}\left(Q^{i, k}, L^{1}\left(\Delta_{i} ; \mathbb{R}_{-}\right)\right)=o\left(\sigma_{k}^{2}\right) .
$$

Then, in addition to A.44, we have proved that

$$
\left\|G_{2}^{+}\left(u^{k}, y^{k}\right)\right\|_{1}=o\left(\sigma_{k}^{2}\right) .
$$

We apply now Lemma A.7 to the sequence $\left(u^{k}, y^{k}\right)$; we get a sequence $\left(\hat{u}^{k}, \hat{y}^{k}\right) \in \mathcal{U}_{\infty} \times \mathcal{Y}_{\infty}$ with $\hat{y}^{k}=\Gamma\left(\hat{u}^{k}, y^{0, k}\right)$ and such that

$$
\begin{aligned}
\left\|\hat{u}^{k}-u^{k}\right\|_{\infty} & =o(1), \\
\left\|\hat{u}^{k}-u^{k}\right\|_{1} & =o\left(\sigma_{k}^{2}\right), \\
\left\|G_{2}^{+}\left(\hat{u}^{k}, \hat{y}^{k}\right)\right\|_{\infty} & =o\left(\sigma_{k}^{2}\right) .
\end{aligned}
$$

Since $G_{1}$ satisfies Property $2,\left(v, z^{0}\right) \in C_{2}^{Q R} A P$ and $\left(w, \xi^{0}\right)$ is feasible for $Q_{\left(v, z^{0}\right)}$, we get

$$
\operatorname{dist}_{X}\left(G_{1}\left(\hat{u}^{k}, y^{0, k}\right), K\right)=o\left(\sigma_{k}^{2}\right),
$$

and then, together with A.58,

$$
\operatorname{dist}_{X_{\infty}}\left(G\left(\hat{u}^{k}, y^{0, k}\right), K_{\infty}\right)=o\left(\sigma_{k}^{2}\right) .
$$

By Robinson's constraint qualification $(\mathrm{A} .9), G$ is metric regular at $\left(\bar{u}, \bar{y}^{0}\right)$ w.r.t. $K_{\infty},[8$, Theorem 2.87]. Then there exists $\left(\tilde{u}^{k}, \tilde{y}^{0, k}\right) \in \mathcal{U}_{\infty} \times \mathbb{R}^{\bar{n}}$ such that

$$
\left\{\begin{array}{l}
\left\|\tilde{u}^{k}-\hat{u}^{k}\right\|_{\infty}+\left|\tilde{y}^{0, k}-y^{0, k}\right|=o\left(\sigma_{k}^{2}\right), \\
G\left(\tilde{u}^{k}, \tilde{y}^{0, k}\right) \in K_{\infty}
\end{array}\right.
$$

Since $\left(\bar{u}, \bar{y}^{0}\right)$ is a locally optimal solution, $J\left(\tilde{u}^{k}, \tilde{y}^{0, k}\right) \geq J\left(\bar{u}, \bar{y}^{0}\right)$ for $k$ big enough. By Property 2 , satisfied by $J$, we have

$$
\sigma_{k} D J\left(\bar{u}, \bar{y}^{0}\right)\left(v, z^{0}\right)+\frac{1}{2} \sigma_{k}^{2}\left(D J\left(\bar{u}, \bar{y}^{0}\right)\left(w, \xi^{0}\right)+D^{2} J\left(\bar{u}, \bar{y}^{0}\right)\left(v, z^{0}\right)^{2}\right)+o\left(\sigma_{k}^{2}\right) \geq 0 .
$$

The conclusion of Lemma A.6 follows by Theorem A.4 and Remark A.3.

\section{A.2 Proof of Proposition 3.8}

The proof of Proposition 3.8 relies on the following two lemmas, proved at the end of the section. The first one is a consequence of Lyapunov theorem [22] and links relaxed dynamics to classical dynamics.

Lemma A.9. Let $F:[0, T] \times \mathbb{R}^{m} \times \mathbb{R}^{n} \rightarrow \mathbb{R}^{n}$ and $G:[0, T] \times \mathbb{R}^{n} \rightarrow \mathbb{R}^{n}$ be uniformly quasi-C $C^{1}$. Let $(\hat{u}, \hat{\alpha}, \hat{y}) \in \mathcal{U} \times \mathcal{A}^{N} \times \mathcal{Y}$ such that, for a.a. $t, 0 \leq \hat{\alpha}^{i} \leq 1 / N$ and

$$
\dot{\hat{y}}_{t}=\left(1-\sum_{i=1}^{N} \hat{\alpha}_{t}^{i}\right) F\left(t, \hat{u}_{t}, \hat{y}_{t}\right)+\sum_{i=1}^{N} \hat{\alpha}_{t}^{i} F\left(t, u_{t}^{i}, \hat{y}_{t}\right)+G\left(t, \hat{y}_{t}\right) .
$$


Then, for any $\varepsilon>0$, there exists $(u, y) \in \mathcal{U} \times \mathcal{Y}$ such that

$$
\begin{aligned}
& \dot{y}_{t}=F\left(t, u_{t}, y_{t}\right)+G\left(t, y_{t}\right) \quad \text { for a.a. } t, \quad y_{0}=\hat{y}_{0}, \\
& u_{t} \in\left\{\hat{u}_{t}, u_{t}^{1}, \ldots, u_{t}^{N}\right\} \quad \text { for a.a. } t, \\
& \|u-\hat{u}\|_{1} \leq \sum_{i=1}^{N}\left\|\hat{\alpha}^{i}\right\|_{1}\left\|u^{i}-\hat{u}\right\|_{\infty}, \\
& \|y-\hat{y}\|_{\infty} \leq \varepsilon .
\end{aligned}
$$

The second one is a metric regularity result, consequence of the qualification of problem $\left(P_{N}\right)$ at $(\bar{u}, \bar{\alpha}, \bar{y}, \bar{\theta})$.

Lemma A.10. There exists $c>0$ such that for any relaxed trajectory $(u, \alpha, y, \theta)$ with $u$ in a $L^{1}$ neighborhood of $\bar{u}$ and $(\alpha, y)$ in a $L^{\infty}$ neighborhood of $(\bar{\alpha}, \bar{y})$, there exists a relaxed trajectory $\left(u^{\prime}, \alpha^{\prime}, y^{\prime}, \theta\right)$ such that

$$
\left\{\begin{array}{l}
\left\|u^{\prime}-u\right\|_{\infty}+\left\|\alpha^{\prime}-\alpha\right\|_{\infty}+\left\|y^{\prime}-y\right\|_{\infty} \leq c\left|\Phi^{E}\left(y_{0}, y_{T}\right)\right|, \\
\Phi^{E}\left(y_{0}^{\prime}, y_{T}^{\prime}\right)=0 .
\end{array}\right.
$$

We can now prove the proposition. The idea is to use alternatively Lemma A.9 to diminish progressively $\hat{\alpha}$, and Lemma A.10 to restore the equality constraints at each step.

Proof of Proposition 3.8. Let $(\hat{u}, \hat{y}, \hat{\alpha}, \hat{\theta}) \in F\left(P_{N}\right)$, close to $(\bar{u}, \bar{y}, \bar{\alpha}, \bar{\theta})$ and with $\hat{\theta}<0$. Without loss of generality, we assume that $\hat{\alpha} \neq 0$ and, see Lemma 3.4 that

$$
c\left(t, u_{t}^{i}, \hat{y}_{t}\right) \leq \hat{\theta} \quad \text { for a.a. } t, \quad 1 \leq i \leq N
$$

Let $R:=\operatorname{diam}_{L^{\infty}}\left\{\hat{u}, u^{1}, \ldots, u^{N}\right\}$ and let $\varepsilon>0$. We claim that there exists a sequence $\left(\hat{u}^{k}, \hat{y}^{k}, \hat{\alpha}^{k}, \hat{\theta}^{k}\right) \in F\left(P_{N}\right)$ such that $\left(\hat{u}^{0}, \hat{y}^{0}, \hat{\alpha}^{0}, \hat{\theta}^{0}\right)=(\hat{u}, \hat{y}, \hat{\alpha}, \hat{\theta})$, and for all $k$,

$$
\begin{aligned}
& \operatorname{diam}_{L^{\infty}}\left\{\hat{u}^{k}, u^{1}, \ldots, u^{N}\right\}<2 R, \\
& c\left(t, u_{t}^{i}, \hat{y}_{t}^{k}\right) \leq \hat{\theta}^{k} \quad \text { for a.a. } t, \quad 1 \leq i \leq N, \\
& \left\|\hat{u}^{k+1}-\hat{u}^{k}\right\|_{1} \leq\left(\frac{3}{4}\right)^{k+1} 2 R N T\|\hat{\alpha}\|_{\infty}, \\
& \left\|\hat{y}^{k+1}-\hat{y}^{k}\right\|_{\infty} \leq\left(\frac{3}{4}\right)^{k+1} \frac{\varepsilon}{4}, \\
& \left\|\hat{\alpha}^{k+1}\right\|_{\infty} \leq\left(\frac{3}{4}\right)^{k+1}\|\hat{\alpha}\|_{\infty}, \\
& \hat{\theta}^{k+1}=\frac{1}{4} \hat{\theta}^{k} .
\end{aligned}
$$

Suppose for a while that we have such a sequence. By $\mathrm{A} .72)-\mathrm{A} .74)$, there exist $\tilde{u} \in L^{1}\left(0, T ; R^{m}\right)$ and $\tilde{y} \in C\left([0, T] ; \mathbb{R}^{n}\right)$, and $\hat{u}^{k} \rightarrow \tilde{u}$ in $L^{1}, \hat{y}^{k} \rightarrow \tilde{y}$ in $C$, and $\hat{\alpha}^{k} \rightarrow 0$ in $L^{\infty}$. By $(\mathrm{A} .70), \tilde{u} \in \mathcal{U}$, and since $\left(\hat{u}^{k}, \hat{y}^{k}, \hat{\alpha}^{k}, \hat{\theta}^{k}\right) \in F\left(P_{N}\right)$ and $\hat{\theta}^{k}<0$ for all $k$, we get that $(\tilde{u}, \tilde{y}) \in F(P)$ by doing $k \rightarrow \infty$ in the relaxed dynamics and in the constraints. Finally,

$$
\|\tilde{u}-\hat{u}\|_{1} \leq 8 R N T\|\hat{\alpha}-\bar{\alpha}\|_{\infty} \quad \text { and } \quad\|\tilde{y}-\hat{y}\|_{\infty} \leq \varepsilon .
$$

It remains to prove the existence the sequence. Suppose we have it up to index $k$ and let us get the next term. Let $F^{k}$ and $G^{k}$ be defined by

$$
F^{k}(t, u, y):=\left(1-\sum_{i=1}^{N} \frac{\hat{\alpha}_{t}^{i, k}}{2}\right) f(t, u, y), \quad G^{k}(t, y):=\sum_{i=1}^{N} \frac{\hat{\alpha}_{t}^{i, k}}{2} f\left(t, u_{t}^{i}, y\right) .
$$

$\mathrm{RR} \mathrm{n}^{\circ} 8306$ 
Since $\left(\hat{u}^{k}, \hat{y}^{k}, \hat{\alpha}^{k}, \hat{\theta}^{k}\right)$ is a relaxed trajectory, we can write

$$
\begin{aligned}
\dot{\hat{y}}_{t}^{k}=\left(1-\sum_{i=1}^{N} \frac{\hat{\alpha}_{t}^{i, k} / 2}{1-\sum_{j=1}^{N} \hat{\alpha}_{t}^{j, k} / 2}\right) F^{k}\left(t, \hat{u}_{t}^{k}, \hat{y}_{t}^{k}\right) & \\
& +\sum_{i=1}^{N} \frac{\hat{\alpha}_{t}^{i, k} / 2}{1-\sum_{j=1}^{N} \hat{\alpha}_{t}^{j, k} / 2} F^{k}\left(t, u_{t}^{i}, \hat{y}_{t}^{k}\right)+G^{k}\left(t, \hat{y}_{t}^{k}\right) .
\end{aligned}
$$

Let $\varepsilon^{\prime}>0$. We apply Lemma A.9 and we get $(u, y) \in \mathcal{U} \times \mathcal{Y}$ such that $\left(u, y, \hat{\alpha}^{k} / 2, \hat{\theta}^{k}\right)$ is a relaxed trajectory, and

$$
\begin{aligned}
& u_{t} \in\left\{\hat{u}_{t}^{k}, u_{t}^{1}, \ldots, u_{t}^{N}\right\} \quad \text { for a.a. } t \\
& \left\|u-\hat{u}^{k}\right\|_{1} \leq \sum_{i=1}^{N}\left\|\frac{\hat{\alpha}_{t}^{k, i} / 2}{1-\sum \hat{\alpha}_{t}^{k, j} / 2}\right\|_{1}\left\|u^{i}-\hat{u}^{k}\right\|_{\infty}, \\
& \left\|y-\hat{y}^{k}\right\|_{\infty} \leq \varepsilon^{\prime} .
\end{aligned}
$$

By $\mathrm{A} .79$, we have

$$
\begin{aligned}
& \operatorname{diam}_{L^{\infty}}\left\{u, u^{1}, \ldots, u^{N}\right\} \leq \operatorname{diam}_{L^{\infty}}\left\{\hat{u}^{k}, u^{1}, \ldots, u^{N}\right\}<2 R, \\
& c\left(t, u_{t}, \hat{y}_{t}^{k}\right) \leq \hat{\theta}^{k} \quad \text { for a.a. } t .
\end{aligned}
$$

By A.81, and since $\hat{\theta}^{k}<0$, we have for $\varepsilon^{\prime}$ small enough,

$$
\begin{aligned}
& c\left(t, u_{t}, y_{t}\right) \leq \frac{1}{2} \hat{\theta}^{k} \quad \text { for a.a. } t, \\
& g\left(t, y_{t}\right) \leq \frac{1}{2} \hat{\theta}^{k} \quad \text { for a.a. } t, \\
& \Phi^{I}\left(y_{0}, y_{T}\right) \leq \frac{1}{2} \hat{\theta}^{k}, \\
& \phi\left(y_{0}, y_{T}\right)-\phi\left(\bar{y}_{0}, \bar{y}_{T}\right) \leq \frac{1}{2} \hat{\theta}^{k}, \\
& \Phi^{E}\left(y_{0}, y_{T}\right)=O\left(\varepsilon^{\prime}\right) .
\end{aligned}
$$

Observe that

$$
\left|1-\sum \hat{\alpha}_{t}^{k, j} / 2\right| \geq 1-N\|\hat{\alpha}\|_{\infty} \geq \frac{3}{4}
$$

for $\|\hat{\alpha}\|_{\infty}$ small enough. Then by A.74, A.80 and A.82,

$$
\left\|u-\hat{u}^{k}\right\|_{1} \leq \frac{3}{8}\left(\frac{3}{4}\right)^{k} 2 R N T\|\hat{\alpha}\|_{\infty}
$$

We now apply Lemma A.10 to $\left(u, y, \hat{\alpha}^{k} / 2\right)$ and we get $\left(\hat{u}^{k+1}, \hat{y}^{k+1}, \hat{\alpha}^{k+1}\right)$ such that $\Phi^{E}\left(\hat{y}_{0}^{k+1}, \hat{y}_{T}^{k+1}\right)=$ 0 and, by A.88,

$$
\left\|\hat{u}^{k+1}-u\right\|_{\infty}+\left\|\hat{y}^{k+1}-y\right\|_{\infty}+\left\|\hat{\alpha}^{k+1}-\frac{\hat{\alpha}^{k}}{2}\right\|_{\infty}=O\left(\varepsilon^{\prime}\right) .
$$


Then for $\hat{\theta}^{k+1}:=\hat{\theta}^{k} / 4$ and $\varepsilon^{\prime}$ small enough, $\left(\hat{u}^{k+1}, \hat{y}^{k+1}, \hat{\alpha}^{k+1}, \hat{\theta}^{k+1}\right) \in F\left(P_{N}\right)$. Moreover,

$$
\begin{aligned}
& \operatorname{diam}_{L^{\infty}}\left\{\hat{u}^{k+1}, u^{1}, \ldots, u^{N}\right\}<2 R+\left\|\hat{u}^{k+1}-u\right\|_{\infty}, \\
& \left\|\hat{u}^{k+1}-\hat{u}^{k}\right\|_{1} \leq \frac{3}{8}\left(\frac{3}{4}\right)^{k} 2 R N T\|\hat{\alpha}\|_{\infty}+T\left\|\hat{u}^{k+1}-u\right\|_{\infty}, \\
& \left\|\hat{y}^{k+1}-\hat{y}^{k}\right\|_{\infty} \leq \varepsilon^{\prime}+\left\|\hat{y}^{k+1}-y\right\|_{\infty}, \\
& \left\|\hat{\alpha}^{k+1}\right\|_{\infty} \leq \frac{1}{2}\left(\frac{3}{4}\right)^{k}\|\hat{\alpha}\|_{\infty}+\left\|\hat{\alpha}^{k+1}-\frac{\hat{\alpha}^{k}}{2}\right\|_{\infty}
\end{aligned}
$$

By A.91), and since $\|\hat{\alpha}\|_{\infty} \neq 0$, we get the sequence up to index $k+1$ for $\varepsilon^{\prime}$ small enough.

Proof of Lemma A.9. We need the following consequence of Gronwall's lemma:

Lemma A.11. Let $B:[0, T] \times \mathbb{R}^{n} \rightarrow \mathbb{R}^{n}$ be uniformly quasi- $C^{1}$. Then there exists $C>0$ such that, for any $b \in L^{\infty}\left(0, T ; \mathbb{R}^{n}\right)$ and $e^{1}, e^{2} \in \mathcal{Y}$ such that

$$
\left\{\begin{array}{l}
\dot{e}_{t}^{2}-\dot{e}_{t}^{1}=B\left(t, e_{t}^{2}\right)-B\left(t, e_{t}^{1}\right)+b_{t} \quad \text { for a.a. } t, \\
e_{0}^{2}-e_{0}^{1}=0
\end{array}\right.
$$

we have

$$
\left\|e^{2}-e^{1}\right\|_{\infty} \leq C\|\hat{b}\|_{1}
$$

where $\hat{b}$ is defined by $\hat{b}_{t}:=\int_{0}^{t} b_{s} \mathrm{~d} s$.

Proof. Let $w:=e^{2}-e^{1}-\hat{b}$. Then $\dot{w}_{t}=B\left(t, e_{t}^{2}\right)-B\left(t, e_{t}^{1}\right)$, and

$$
\left|\dot{w}_{t}\right| \leq C^{\prime}\left|e_{t}^{2}-e_{t}^{1}\right| \leq C^{\prime}\left(\left|w_{t}\right|+\left|\hat{b}_{t}\right|\right) .
$$

The result follows by Gronwall's lemma.

Let $\varepsilon>0, M \in \mathbb{N}^{*}$, and $t_{j}:=j T / M$ for $0 \leq j \leq M$. Let us denote by $\left(e_{i}\right)_{i}, 1 \leq i \leq N$ the canonical basis of $\mathbb{R}^{N}$, and let us define $\widetilde{F}^{i}:[0, T] \rightarrow \mathbb{R}^{n} \times \mathbb{R}^{N}$ by

$$
\widetilde{F}_{t}^{0}:=\left(F\left(t, \hat{u}_{t}, \hat{y}_{t}\right), 0\right), \quad \tilde{F}_{t}^{i}:=\left(F\left(t, u_{t}^{i}, \hat{y}_{t}\right), e_{i}\right) \quad 1 \leq i \leq N .
$$

For $0 \leq j<M$, we apply Lyapunov theorem 22 to the family $\left(\tilde{F}^{i}\right)_{i}$ with coefficients $\left(\hat{\alpha}^{i}\right)_{i}$ on $\left[t_{j}, t_{j+1}\right]$. We get the existence of $\alpha \in \mathcal{A}^{N}$, with values in $\{0,1\}^{N}$, and such that for $0 \leq j<M$,

$$
\int_{t_{j}}^{t_{j+1}}\left[\left(1-\sum_{i=1}^{N} \alpha_{t}^{i}\right) \tilde{F}_{t}^{0}+\sum_{i=1}^{N} \alpha_{t}^{i} \tilde{F}_{t}^{i}\right] \mathrm{d} t=\int_{t_{j}}^{t_{j+1}}\left[\left(1-\sum_{i=1}^{N} \hat{\alpha}_{t}^{i}\right) \tilde{F}_{t}^{0}+\sum_{i=1}^{N} \hat{\alpha}_{t}^{i} \tilde{F}_{t}^{i}\right] \mathrm{d} t .
$$

Projecting A.100 on the first $n$ coordinates, we get that

$$
\begin{aligned}
\int_{t_{j}}^{t_{j+1}}\left[\left(1-\sum_{i=1}^{N} \alpha_{t}^{i}\right) F\left(t, \hat{u}_{t}, \hat{y}_{t}\right)+\sum_{i=1}^{N} \alpha_{t}^{i} F\left(t, u_{t}^{i}, \hat{y}_{t}\right)\right] \mathrm{d} t \\
=\int_{t_{j}}^{t_{j+1}}\left[\left(1-\sum_{i=1}^{N} \hat{\alpha}_{t}^{i}\right) F\left(t, \hat{u}_{t}, \hat{y}_{t}\right)+\sum_{i=1}^{N} \hat{\alpha}_{t}^{i} F\left(t, u_{t}^{i}, \hat{y}_{t}\right)\right] \mathrm{d} t .
\end{aligned}
$$

RR n 8306 
Let $u_{t}:=\hat{u}_{t}+\sum_{i=1}^{N} \alpha_{t}^{i}\left(u_{t}^{i}-\hat{u}_{t}\right)$. Note that for a.a. $t, u_{t} \in\left\{\hat{u}_{t}, \ldots, u_{t}^{N}\right\}$. We get by A.101 that

$$
\int_{t_{j}}^{t_{j+1}} F\left(t, u_{t}, \hat{y}_{t}\right) \mathrm{d} t=\int_{t_{j}}^{t_{j+1}}\left[\left(1-\sum_{i=1}^{N} \hat{\alpha}_{t}^{i}\right) F\left(t, \hat{u}_{t}, \hat{y}_{t}\right)+\sum_{i=1}^{N} \hat{\alpha}_{t}^{i} F\left(t, u_{t}^{i}, \hat{y}_{t}\right)\right] \mathrm{d} t .
$$

Projecting A.100 on the last $N$ coordinates, we get that for $1 \leq i \leq N$,

$$
\int_{t_{j}}^{t_{j+1}} \alpha_{t}^{i} \mathrm{~d} t=\int_{t_{j}}^{t_{j+1}} \hat{\alpha}_{t}^{i} \mathrm{~d} t
$$

Summing A.103 for $0 \leq j \leq M$, we get that $\left\|\alpha^{i}\right\|_{1}=\left\|\hat{\alpha}^{i}\right\|_{1}$ for $1 \leq i \leq N$. Since

$$
\|u-\hat{u}\|_{1} \leq \sum_{i=1}^{N}\|\alpha\|_{1}\left\|u^{i}-\hat{u}\right\|_{\infty}
$$

we get A.66). Let $y$ be the unique solution of A.64); we estimate $\|y-\hat{y}\|_{\infty}$ with Lemma A.11. Let $b$ be defined by

$$
b_{t}:=F\left(t, u_{t}, \hat{y}_{t}\right)-\left(1-\sum_{i=1}^{N} \hat{\alpha}_{t}^{i}\right) F\left(t, \hat{u}_{t}, \hat{y}_{t}\right)-\sum_{i=1}^{N} \hat{\alpha}_{t}^{i} F\left(t, u_{t}^{i}, \hat{y}_{t}\right)
$$

and let $\hat{b}$ be defined by $\hat{b}_{t}:=\int_{0}^{t} b_{s} \mathrm{~d} s$. By A.102, $\hat{b}_{t_{j}}=0$ for $0 \leq j \leq M$. Therefore, $\|\hat{b}\|_{\infty}=$ $O(1 / M)$. Observe now that for a.a. $t$,

$$
\dot{y}_{t}-\dot{\hat{y}}_{t}=F\left(t, u_{t}, y_{t}\right)+G\left(t, y_{t}\right)-F\left(t, u_{t}, \hat{y}_{t}\right)-G\left(t, \hat{y}_{t}\right)+b_{t} .
$$

By Lemma A.11. $\|y-\hat{y}\|_{\infty}=O(1 / M)$. For $M$ large enough, we get A.67), and the proof is completed.

Proof of Lemma A.10. Note that the $L^{1}$-distance is involved for the control. The lemma is obtained with an extension of the nonlinear open mapping theorem [1, Theorem 5]. This result can be applied since the derivative of the mapping defined in (3.21) can be described explicitely with a linearized state equation and therefore, by Gronwall's lemma, is continuous for the $L^{1}$-distance on the control $u$.

\section{A.3 A qualification condition}

\section{A.3.1 Statement}

We give here a qualification condition equivalent to the non singularity of generalized Pontryagin multipliers. This qualification condition is expressed with the Pontryagin linearization [24, Proposition 8.1]. In this section, $(\bar{u}, \bar{y}) \in F(P)$ is given. We will always assume that Assumption 2 holds.

Definition A.12. We say that $\lambda=(\beta, \Psi, \nu, \mu) \in \Lambda_{L}(\bar{u}, \bar{y})$ is singular iff $\beta=0$ and that $\lambda$ is normal iff $\beta=1$.

Given $u \in \mathcal{U}$, we define the Pontryagin linearization $\xi[u] \in \mathcal{Y}$ as the unique solution of

$$
\left\{\begin{array}{l}
\dot{\xi}_{t}[u]=D_{y} f\left(t, \bar{u}_{t}, \bar{y}_{t}\right) \xi_{t}[u]+f\left(t, u_{t}, \bar{y}_{t}\right)-f\left(t, \bar{u}_{t}, \bar{y}_{t}\right), \\
\xi_{0}[u]=0 .
\end{array}\right.
$$

Note that $\xi[\bar{u}]=0$. Recall that $U$ is the set-valued mapping defined by 2.22 . We define

$$
\mathcal{U}_{c}:=\left\{u \in \mathcal{U}: u_{t} \in U(t) \text { for a.a. } t\right\} .
$$


Definition A.13. We say that the problem is qualified in the Pontryagin sense (in short Pqualified) at $(\bar{u}, \bar{y})$ iff

(i) the following surjectivity condition holds:

$$
0 \in \operatorname{int}\left\{D \Phi^{E}\left(\bar{y}_{0}, \bar{y}_{T}\right)\left(z_{0}, \xi_{T}[u]+z_{T}\left[v, z_{0}\right]\right): u \in \mathcal{U}_{c}, v \in \mathcal{U}, z_{0} \in \mathbb{R}^{n}\right\}
$$

(ii) there exist $\varepsilon>0, \hat{u} \in \mathcal{U}_{c}, \hat{v} \in \mathcal{U}$, and $\hat{z}_{0} \in \mathbb{R}^{n}$ such that

$$
D \Phi^{E}\left(\bar{y}_{0}, \bar{y}_{T}\right)\left(\hat{z}_{0}, \xi_{T}[\hat{u}]+z_{T}\left[\hat{v}, \hat{z}_{0}\right]\right)=0,
$$

and for a.a. $t$,

$$
\left\{\begin{array}{l}
\Phi^{I}\left(\bar{y}_{0}, \bar{y}_{T}\right)+D \Phi^{I}\left(\bar{y}_{0}, \bar{y}_{T}\right)\left(\hat{z}_{0}, \xi_{T}[\hat{u}]+z_{T}\left[\hat{v}, \hat{z}_{0}\right]\right) \leq-\varepsilon \\
g\left(t, \bar{y}_{t}\right)+D g\left(t, \bar{y}_{t}\right)\left(\xi_{t}[\hat{u}]+z_{t}\left[\hat{v}, \hat{z}_{0}\right]\right) \leq-\varepsilon \\
c\left(t, \bar{u}_{t}, \bar{y}_{t}\right)+D_{y} c\left(t, \bar{u}_{t}, \bar{y}_{t}\right) \xi_{t}[\hat{u}]+D c\left(t, \bar{u}_{t}, \bar{y}_{t}\right)\left(\hat{v}_{t}, z_{t}\left[\hat{v}_{t}, \hat{z}_{0}\right]\right) \leq-\varepsilon .
\end{array}\right.
$$

Note that if we impose $u=\bar{u}$ in the definition of the P-qualification, we obtain the usual qualification conditions, which are equivalent to the normality of Lagrange multipliers. The Pqualification is then weaker, and as proved in the next theorem, it is necessary and sufficient to ensure the non singularity of Pontryagin multipliers.

Theorem A.14. Let Assumption 2 hold. Then, the set of singular Pontryagin multipliers is empty if and only if the problem is $P$-qualified.

We prove this result in the following two paragraphs.

Proposition A.15. Let Assumption 2 hold. If the set of singular Pontryagin multipliers is empty, then the set of normal Pontryagin multipliers is bounded in E.

Proof. Remember that the norm of $E$ is defined by (2.11). We prove the result by contraposition and consider a sequence $\left(\lambda^{k}\right)_{k}$ of normal Pontryagin multipliers which is such that $\left\|\lambda^{k}\right\|_{E} \rightarrow+\infty$. Then, by Lemma 3.5 the sequence $\lambda^{k} /\left\|\lambda^{k}\right\|_{E}$ possesses a weak limit point in $\Lambda_{P}(\bar{u}, \bar{y})$, say $\lambda=(\beta, \Psi, \nu, \mu)$, which is such that

$$
\beta=\lim _{k} \frac{1}{\left\|\lambda^{k}\right\|_{E}}=0 .
$$

Therefore, $\lambda$ is singular. The proposition is proved.

\section{A.3.2 Sufficiency of the qualification condition}

In this paragraph, we prove by contradiction that the $\mathrm{P}$-qualification implies the non singularity of Pontryagin multipliers. Let us assume that the problem is P-qualified and that there exists $\lambda=$ $(\beta, \Psi, \nu, \mu) \in \Lambda_{P}(\bar{u}, \bar{y})$ with $\beta=0$ and $\Psi=\left(\Psi^{E}, \Psi^{I}\right)$. Let $\hat{u}, \hat{w}, \hat{z}_{0}$ be such that A.110 - A.111 hold. With an integration by parts and using the stationarity of the augmented Hamiltonian, we get that for all $u \in \mathcal{U}_{c}, v \in \mathcal{U}$, and $z_{0} \in \mathbb{R}^{n}$,

$$
\begin{gathered}
\int_{0}^{T} \nu_{t}\left(D c\left(t, \bar{u}_{t}, \bar{y}_{t}\right)\left(v_{t}, z_{t}\left[v, z_{0}\right]\right)+D_{y} c\left(t, \bar{u}_{t}, \bar{y}_{t}\right) \xi_{t}[u]\right) \mathrm{d} t \\
\quad+\int_{0}^{T} D g\left(t, \bar{y}_{t}\right)\left(\xi_{t}[u]+z_{t}\left[v, z_{0}\right]\right) \mathrm{d} \mu_{t} \\
\quad+D \Phi\left[0,\left(\Psi^{E}, \Psi^{I}\right)\right]\left(\bar{y}_{0}, \bar{y}_{T}\right)\left(z_{0}, \xi_{T}[u]+z_{T}\left[v, z_{0}\right]\right) \\
=\int_{0}^{T} H\left[p_{t}^{\lambda}\right]\left(t, u_{t}, \bar{y}_{t}\right)-H\left[p_{t}^{\lambda}\right]\left(t, \bar{u}_{t}, \bar{y}_{t}\right) \mathrm{d} t \geq 0 .
\end{gathered}
$$

$\mathrm{RR} \mathrm{n}^{\circ} 8306$ 
By A.110-A.111 and the nonnegativity of $\Psi^{I}, \nu$, and $\mu$, we obtain that for $u=\hat{u}, v=\hat{v}$, $z_{0}=\hat{z}_{0}$, the r.h.s. of A.113 is nonpositive and thus equal to 0 . Therefore, $\Psi^{I}, \nu$, and $\mu$ are null and for all $u \in \mathcal{U}_{c}, v \in \mathcal{U}$, and $z_{0} \in \mathbb{R}^{n}$,

$$
\Psi^{E} D \Phi^{E}\left(\bar{y}_{0}, \bar{y}_{T}\right)\left(z_{0}, \xi_{T}[u]+z_{T}\left[v, z_{0}\right]\right) \geq 0 .
$$

By A.109, we can choose $u, v$, and $z_{0}$ so that for $\beta>0$ sufficiently small,

$$
D \Phi^{E}\left(\bar{y}_{0}, \bar{y}_{T}\right)\left(z_{0}, \xi_{T}[u]+z_{T}\left[v, z_{0}\right]\right)=-\beta\left(\Psi^{E}\right)^{T} .
$$

Combined with A.114, we obtain that $-\beta\left|\Psi^{E}\right|^{2} \geq 0$. Then, $\Psi^{E}=0$ and finally $\lambda=0$, in contradiction with $\lambda \in \Lambda_{P}(\bar{u}, \bar{y})$.

\section{A.3.3 Necessity of the qualification condition}

We now prove that the P-qualification is necessary to ensure the non singularity of Pontryagin multipliers. In some sense, the approach consists in describing this qualification condition as the limit of the qualification conditions associated with a sequence of partially relaxed problems.

Let us fix a Castaing representation $\left(u^{k}\right)_{k}$ of $U$. For all $N \in \mathbb{N}$, we consider a partially relaxed problem $\left[\tilde{P}_{N}\right)$ defined by

$$
\min _{u \in \mathcal{U}, \alpha \in \mathcal{A}^{N}, y \in \mathcal{Y}} \phi\left(y_{0}, y_{T}\right) \quad \text { s.t. constraints 2.3 } 2.5, y=y\left[u, \alpha, y_{0}\right] \text {, and } \alpha \geq 0
$$

where $y\left[u, \alpha, y^{0}\right]$ is the solution to the partially relaxed state equation 3.12 . This problem is the same as problem $\left(P_{N}\right)$, except that there is no variable $\theta$.

For given $v \in \mathcal{U}, z_{0} \in \mathbb{R}^{n}$ and $\alpha \in \mathcal{A}^{N}$, we denote by $z\left[v, z_{0}\right]$ the linearized state variable in the direction $\left(v, z_{0}\right)$, which is the solution to $(4.2)$ and we denote by $\xi[\alpha]$ the linearized state variable in the direction $\alpha$, which is the solution to

$$
\left\{\begin{array}{l}
\dot{\xi}_{t}[\alpha]=D_{y} f\left(t, \bar{u}_{t}, \bar{y}_{t}\right) \xi_{t}[\alpha]+\sum_{i=1}^{N} \alpha_{t}^{i}\left(f\left(t, u_{t}^{i}, \bar{y}_{t}\right)-f\left(t, \bar{u}_{t}, \bar{y}_{t}\right)\right), \\
\xi_{0}[\alpha]=0
\end{array}\right.
$$

The distinction between the Pontryagin linearization $\xi[u]$ and $\xi[\alpha]$ will be clear in the sequel, and we will motivate this choice of notations in Lemma A.18.

Problem $\left[\tilde{P}_{N}\right.$ is qualified (in the usual sense) iff

(i) the following surjectivity condition holds:

$$
0 \in \operatorname{int}\left\{D \Phi^{E}\left(\bar{y}_{0}, \bar{y}_{T}\right)\left(z_{0}, \xi_{T}[\alpha]+z_{T}\left[v, z_{0}\right]\right): \alpha \in \mathcal{A}^{N}, v \in \mathcal{U}, z_{0} \in \mathbb{R}^{n}\right\}
$$

(ii) there exist $\varepsilon>0, \hat{\alpha} \in \mathcal{A}^{N}, \hat{v} \in \mathcal{U}, \hat{z}_{0} \in \mathbb{R}^{n}$ such that

$$
D \Phi^{E}\left(\bar{y}_{0}, \bar{y}_{T}\right)\left(\hat{z}_{0}, \xi_{T}[\hat{\alpha}]+z_{T}\left[\hat{v}, \hat{z}_{0}\right]\right)=0
$$

and

$$
\left\{\begin{array}{l}
\Phi^{I}\left(\bar{y}_{0}, \bar{y}_{T}\right)+D \Phi^{I}\left(\bar{y}_{0}, \bar{y}_{T}\right)\left(\hat{z}_{0}, \xi_{T}[\hat{\alpha}]+z_{T}\left[\hat{v}, \hat{z}_{0}\right]\right) \leq-\varepsilon \\
g\left(t, \bar{y}_{t}\right)+D g\left(t, \bar{y}_{t}\right)\left(\xi_{t}[\hat{\alpha}]+z_{t}\left[\hat{v}, \hat{z}_{0}\right]\right) \leq-\varepsilon, \quad \text { for all } t \\
c\left(t, \bar{u}_{t}, \bar{y}_{t}\right)+D c\left(t, \bar{u}_{t}, \bar{y}_{t}\right)\left(\hat{v}_{t}, \xi_{t}[\hat{\alpha}]+z_{t}\left[\hat{v}, \hat{z}_{0}\right]\right) \leq-\varepsilon, \quad \text { for a.a. } t \\
\hat{\alpha}_{t} \geq \varepsilon, \quad \text { for a.a. } t
\end{array}\right.
$$


We denote now by $\Lambda\left(\tilde{P}_{N}\right)$ the set of generalized Lagrange multipliers of problem $\left[\tilde{P}_{N}\right]$ at $(\bar{u}, \alpha=0, \bar{y})$. Following the proof of Lemma 3.10 we easily obtain that

$$
\begin{aligned}
\Lambda\left(\tilde{P}_{N}\right)=\left\{(\lambda, \gamma) \in \Lambda^{N}(\bar{u}, \bar{y}) \times L^{\infty}\left([0, T] ; \mathbb{R}_{+}^{k}\right):\right. \\
\left.\gamma_{t}^{i}=H\left[p_{t}^{\lambda}\right]\left(t, u_{t}^{i}, \bar{y}_{t}\right)-H\left[p_{t}^{\lambda}\right]\left(t, \bar{u}_{t}, \bar{y}_{t}\right), \text { for } i=1, \ldots, N, \text { for a.a. } t\right\},
\end{aligned}
$$

where $\Lambda^{N}(\bar{u}, \bar{y})$ is defined by 3.5 and $\gamma$ is associated with the constraint $\alpha \geq 0$.

Lemma A.16. Let $N \in \mathbb{N}$; all multipliers of $\Lambda^{N}(\bar{u}, \bar{y})$ are non singular if and only if problem $\tilde{P}_{N}$ is qualified.

Proof. It is known that all multipliers of $\Lambda\left(\tilde{P}_{N}\right)$ are non singular if and only if problem $\left(\tilde{P}_{N}\right)$ is qualified, see e.g. [8, Proposition 3.16]. It follows from (A.120) that all multipliers of $\Lambda^{N}(\bar{u}, \bar{y})$ are non singular if and only if the multipliers of $\Lambda\left(\tilde{P}_{N}\right)$ are non singular. This proves the lemma.

As a corollary, we obtain that if problem $\left(\tilde{P}_{N}\right)$ is qualified at stage $N$, it is also qualified at stage $N+1$. Indeed, if none of the multipliers in $\Lambda^{N}(\bar{u}, \bar{y})$ is singular, a fortiori, none of the multipliers in $\Lambda^{N+1}(\bar{u}, \bar{y})$ is singular, since $\Lambda^{N+1}(\bar{u}, \bar{y}) \subset \Lambda^{N}(\bar{u}, \bar{y})$.

Proposition A.17. The set of singular Pontryagin multipliers is empty if and only if there exists $N \in \mathbb{N}$ such that problem $\sqrt{\left.\tilde{P}_{N}\right)}$ is qualified.

Proof. Let $N \in \mathbb{N}$ be such that problem $\left(\tilde{P}_{N}\right]$ is qualified. Then, all multipliers of $\Lambda^{N}(\bar{u}, \bar{y})$ are non singular, by Lemma A.16. Since $\Lambda_{P}(\bar{u}, \bar{y}) \subset \Lambda^{N}(\bar{u}, \bar{y})$, the Pontryagin multipliers are non singular.

Conversely, assume that for all $N \in \mathbb{N}$, problem $\sqrt{\tilde{P}_{N}}$ is not qualified. By Lemma A.16, we obtain a sequence of singular multipliers $\left(\lambda^{N}\right)_{N}$ which is such that for all $N, \lambda^{N} \in \Lambda^{N}(\bar{u}, \bar{y})$. Normalizing this sequence, we obtain with Lemma 3.5 the existence of a weak limit point in $\Lambda_{P}(\bar{u}, \bar{y})$, which is necessarily singular.

To conclude the proof, we still need a relaxation result, which makes a link between the Pontryagin linearization $\xi[u]$ and the linearization $\xi[\alpha]$.

Lemma A.18. Let $N \in \mathbb{N}$; assume that problem $\tilde{P}_{N}$ is qualified. Then, there exists $A>0$ such that for all $\left(\alpha, v, z_{0}\right) \in \mathcal{A}^{N} \times \mathcal{U}, \mathbb{R}^{n}$ with $\|\alpha\|_{\infty} \leq A,\|v\|_{\infty} \leq A,\left|z_{0}\right| \leq A$, for all $\varepsilon>0$, if $\alpha$ is uniformly positive, then there exists $\left(u, v^{\prime}, z_{0}^{\prime}\right) \in \mathcal{U}_{c} \times \mathcal{U} \times \mathbb{R}^{n}$ such that

$$
\begin{aligned}
& D \Phi^{E}\left(\bar{y}_{0}, \bar{y}_{T}\right)\left(z_{0}, \xi_{T}[u]+z_{T}\left[v, z_{0}\right]\right)=D \Phi^{E}\left(\bar{y}_{0}, \bar{y}_{T}\right)\left(z_{0}, \xi_{T}[\alpha]+z_{T}\left[v, z_{0}\right]\right), \\
& \left\|\xi[u]-\xi[\alpha]+z\left[v^{\prime}-v, z_{0}-z_{0}^{\prime}\right]\right\|_{\infty} \leq \varepsilon .
\end{aligned}
$$

Proof. We only give some elements of proof. Note that this result is a variant of Proposition 3.8 and can be obtained with Dmitruk's result [13, Theorem 3]. Let us define

$$
g(t, u, y):=D_{y} f\left(t, \bar{u}_{t}, \bar{y}_{t}\right) y+f\left(t, u, \bar{y}_{t}\right)-f\left(t, \bar{u}_{t}, \bar{y}_{t}\right) .
$$

Then, for all $u \in \mathcal{U}_{c}, \xi[u]$ is the solution to

$$
\dot{\xi}_{t}[u]=g\left(t, \xi_{t}[u], u_{t}\right), \quad \xi_{0}[u]=0 .
$$

and $\xi[\alpha]$, where $\alpha \in \mathcal{A}^{N}$ and $\alpha \geq 0$ is the solution to the relaxed system associated with the dynamics $g$ and the Castaing representation. Indeed,

$$
\begin{aligned}
\dot{\xi}_{t}[\alpha] & =D_{y} f\left(t, \bar{u}_{t}, \bar{y}_{t}\right) \xi_{t}[\alpha]+\sum_{i=1}^{N} \alpha_{t}^{i}\left(f\left(t, u_{t}^{i}, \bar{y}_{t}\right)-f\left(t, \bar{u}_{t}, \bar{y}_{t}\right)\right) \\
& =\left(1-\sum_{i=1}^{N} \alpha_{t}^{i}\right) g\left(t, \bar{u}_{t}, \bar{y}_{t}\right)+\sum_{i=1}^{N} \alpha_{t}^{i}\left(g\left(t, u_{t}^{i}, \bar{y}_{t}\right)-g\left(t, \bar{u}_{t}, \bar{y}_{t}\right)\right) .
\end{aligned}
$$

$\mathrm{RR} \mathrm{n}^{\circ} 8306$ 
Finally, we prove the result by building a sequence $\left(u^{k}, \alpha^{k}, v^{k}, z_{0}^{k}\right)$ which is such that

$$
\begin{aligned}
& \left(u^{0}, \alpha^{0}, v^{0}, z_{0}^{0}\right)=\left(\bar{u}, \alpha, v, z_{0}\right), \\
& D \Phi^{E}\left(\bar{y}_{0}, \bar{y}_{T}\right)\left(z_{0}, \xi_{T}[\alpha]+z_{T}\left[v, z_{0}\right]\right) \\
& \quad=D \Phi^{E}\left(\bar{y}_{0}, \bar{y}_{T}\right)\left(z_{0}^{k}, \xi_{T}\left[\alpha^{k}\right]+\xi_{T}\left[u^{k}\right]+z_{T}\left[v, z_{0}\right]\right),
\end{aligned}
$$

such that $\alpha^{k}$ is uniformly positive and finally which is such that $\left(u^{k}\right)_{k}$ converges to some $u \in \mathcal{U}_{c}$ in $L^{1}$ norm, $\left(\alpha^{k}\right)_{k}$ converges to 0 in $L^{\infty}$ norm, and $\left(v^{k}, z_{0}^{k}\right)_{k}$ equally converges to some $\left(v^{\prime}, z_{0}^{\prime}\right)$ in $L^{\infty}$ norm. This sequence is built by using Lemma A.9 and by using the surjectivity condition A.118. Note that Lemma A.9 enables to ensure A.122.

Let us conclude the proof of Theorem A.14. Let us assume that the set of singular Pontryagin multipliers is empty; we already know by Proposition A.17 that there exists $N \in \mathbb{N}$ such that the $\mathrm{MF}_{N}$ conditions hold. It remains to prove that the problem is $\mathrm{P}$-qualified. Let $\left(\alpha^{k}, v^{k}, z_{0}^{k}\right)_{k=1, \ldots, n_{\Phi}+1}$ be such that

$$
0 \in \operatorname{int}\left\{\operatorname{conv}\left[D \Phi^{E}\left(\bar{y}_{0}, \bar{y}_{T}\right)\left(z_{0}^{k}, z_{T}\left[v^{k}, z_{0}^{k}\right]+\xi_{T}\left[\alpha^{k}\right]\right), k=1, \ldots, n_{\phi^{E}}+1\right]\right\} .
$$

Let $\left(\hat{\alpha}, \hat{v}, \hat{z}_{0}\right)$ be such that A.119 holds. By A.118, if we replace $\left(\alpha^{k}, v^{k}, z_{0}^{k}\right)$ by $\left(\alpha^{k}+\delta \hat{\alpha}, v^{k}+\right.$ $\left.\delta \hat{v}, z_{0}^{k}+\delta \hat{z}_{0}\right)$, for any $\delta>0$, then A.128 still holds. Moreover, A.128 remains true if we multiply this family by a given positive constant. Therefore, since $\hat{\alpha}$ is uniformly positive, we may assume that the family $\left(\alpha^{k}, v^{k}, z_{0}^{k}\right)_{k=1, \ldots, n_{\phi}{ }+1}$ is bounded by $A$ and such that for all $k=1, \ldots, n_{\Phi^{E}}+1, \alpha^{k}$ is uniformly positive. Finally, we can apply Lemma A.18 to any convex combination of elements of the family. This proves the part of the P-qualification associated with equality constraints. Multiplying $\left(\hat{\alpha}, \hat{v}, \hat{z}_{0}\right)$ by a positive constant, we can assume that it is bounded by $A$ and we can equally approximate it so that A.110 holds and so that A.111) holds (if the variable $\varepsilon$ of Lemma A.18 is chosen sufficiently small). We have proved that the problem was $\mathrm{P}$-qualified.

\section{A.4 An example about Pontryagin's principle}

We give here an example where there exists a multiplier such that the Hamiltonian inequality (2.26) holds for all $u \in U(t)$, but not for all $u$ in

$$
\tilde{U}(t):=\left\{u \in \mathbb{R}^{m}: c\left(t, u, \bar{y}_{t}\right) \leq 0\right\}
$$

Indeed, $U(t) \subset \tilde{U}(t)$ but it may happen that $U(t) \neq \tilde{U}(t)$.

Consider the optimal control problem

$$
\min y_{T}
$$

subject to the following state equation with fixed initial state, in $\mathbb{R}$ :

$$
\dot{y}_{t}=u_{t}, \quad y_{0}=y^{0},
$$

and to the following mixed constraint:

$$
u_{t} \geq-y_{t}, \text { for a.a. } t .
$$

The optimal control $(\bar{u}, \bar{y})$ is such that $\bar{u}_{t}=-\bar{y}_{t}$ and given an initial state $y^{0}$, the optimal solution is given by:

$$
\bar{u}_{t}=-y^{0} e^{-t}, \quad \bar{y}_{t}=y^{0} e^{-t} .
$$


The problem being qualified, there exists a normal Lagrange multiplier which is determined by $\nu$. Since the augmented Hamiltonian is stationary, we obtain that for a.a. $t, p_{t}^{\nu}=\nu_{t}$, and therefore the costate equation writes

$$
-\dot{p}_{t}^{\nu}=-p_{t}^{\nu}, \quad p_{T}^{\nu}=1,
$$

i.e. $p_{t}=\nu_{t}=e^{-(T-t)}>0$. Let us fix $y^{0}=0$, the optimal solution is $(0,0)$ and $\tilde{U}(t)=U(t)=\mathbb{R}_{+}$. The Hamiltonian $p u$ is minimized for a.a. $t$ by $\bar{u}_{t}=0$ since $p_{t}>0$.

Now let us consider a variant of this problem. We replace the previous mixed constraint by the following one:

$$
\psi\left(u_{t}\right) \geq-y_{t},
$$

where $\psi$ is a smooth function such that:

$$
\left\{\begin{array}{l}
\forall u \geq 0, \psi(u)=u, \\
\forall u<0, \psi(u) \leq 0 \text { and } \psi(u)=0 \Longleftrightarrow u=-1 .
\end{array}\right.
$$

For $y^{0}=0,(0,0)$ remains a feasible trajectory, since $\tilde{U}(t)=\mathbb{R}_{+} \cup\{-1\}$. In this case, $U(t)=\mathbb{R}_{+}$. Let us check that $(0,0)$ is still an optimal solution. Let us suppose that there exist a feasible trajectory $(u, y)$ which is such that $y_{T}<0$. Then, let $t \in(0, T)$ be such that

$$
y_{t} \in\left(y_{T}, 0\right) \text { and } \forall s \in[t, T], y_{s} \leq y_{t} .
$$

It follows that for a.a. $s \in(t, T)$,

$$
\psi\left(u_{s}\right) \geq-y_{s}>0 .
$$

Therefore, $u_{s}>0$ and $y$ is nondecreasing on $[t, T]$, in contradiction with $y_{t}>y_{T}$. We have proved that $(0,0)$ is an optimal solution, and the multiplier and costate remain unchanged. However, the minimum of the Hamiltonian over $\tilde{U}(t)$ is reached for

$$
u=-1 \neq \bar{u}_{t} .
$$

\section{References}

[1] F. Álvarez, J. Bolte, J. F. Bonnans, and F. J. Silva. Asymptotic expansions for interior penalty solutions of control constrained linear-quadratic problems. Math. Program., 135(12, Ser. A):473-507, 2012.

[2] L. Ambrosio, N. Fusco, and D. Pallara. Functions of bounded variation and free discontinuity problems. Oxford Mathematical Monographs. The Clarendon Press Oxford University Press, New York, 2000.

[3] J. F. Bonnans, C. de la Vega, and X. Dupuis. First- and second-order optimality conditions for optimal control problems of state constrained integral equations. Journal of Optimization Theory and Applications, Online First, 2013.

[4] J. F. Bonnans, X. Dupuis, and L. Pfeiffer. Second-order sufficient conditions for strong solutions to optimal control problems. Inria Research Report RR-3807, INRIA, May 2013.

[5] J. F. Bonnans and A. Hermant. No-gap second-order optimality conditions for optimal control problems with a single state constraint and control. Math. Program., 117(1-2, Ser. B):21-50, 2009 . 
[6] J. F. Bonnans and A. Hermant. Second-order analysis for optimal control problems with pure state constraints and mixed control-state constraints. Ann. Inst. H. Poincaré Anal. Non Linéaire, 26(2):561-598, 2009.

[7] J. F. Bonnans and N. P. Osmolovskiı̌. Second-order analysis of optimal control problems with control and initial-final state constraints. J. Convex Anal., 17(3-4):885-913, 2010.

[8] J. F. Bonnans and A. Shapiro. Perturbation analysis of optimization problems. Springer Series in Operations Research. Springer-Verlag, New York, 2000.

[9] C. Castaing. Sur les multi-applications mesurables. Rev. Française Informat. Recherche Opérationnelle, 1(1):91-126, 1967.

[10] C. Castaing and M. Valadier. Convex analysis and measurable multifunctions. Lecture Notes in Mathematics, Vol. 580. Springer-Verlag, Berlin, 1977.

[11] R. Cominetti. Metric regularity, tangent sets, and second-order optimality conditions. Appl. Math. Optim., 21(3):265-287, 1990.

[12] A. V. Dmitruk. Maximum principle for the general optimal control problem with phase and regular mixed constraints. Comput. Math. Model., 4(4):364-377, 1993. Software and models of systems analysis. Optimal control of dynamical systems.

[13] A. V. Dmitruk. An approximation theorem for a nonlinear control system with sliding modes. Tr. Mat. Inst. Steklova, 256 (Din. Sist. i Optim.):102-114, 2007.

[14] A. V. Dmitruk. Jacobi type conditions for singular extremals. Control Cybernet., 37(2):285$306,2008$.

[15] A. Ja. Dubovickiĭ and A. A. Miljutin. Extremal problems with constraints. Ž. Vyčisl. Mat. i Mat. Fiz., 5:395-453, 1965.

[16] A. Ja. Dubovickiǐ and A. A. Miljutin. Necessary conditions for a weak extremum in optimal control problems with mixed constraints of inequality type. Z̆. Vyčisl. Mat. i Mat. Fiz., 8:725-779, 1968.

[17] R. V. Gamkrelidze. On sliding optimal states. Dokl. Akad. Nauk SSSR, 143:1243-1245, 1962.

[18] R. F. Hartl, S. P. Sethi, and R. G. Vickson. A survey of the maximum principles for optimal control problems with state constraints. SIAM Rev., 37(2):181-218, 1995.

[19] M. R. Hestenes. Calculus of variations and optimal control theory. John Wiley \& Sons Inc., New York, 1966.

[20] R. P. Hettich and H. Th. Jongen. Semi-infinite programming: conditions of optimality and applications. In Optimization techniques (Proc. 8th IFIP Conf., Würzburg, 1977), Part 2, pages 1-11. Lecture Notes in Control and Information Sci., Vol. 7. Springer, Berlin, 1978.

[21] H. Kawasaki. An envelope-like effect of infinitely many inequality constraints on secondorder necessary conditions for minimization problems. Math. Programming, 41(1, (Ser. A)):73-96, 1988.

[22] A. Liapounoff. Sur les fonctions-vecteurs complètement additives. Bull. Acad. Sci. URSS. Sér. Math. [Izvestia Akad. Nauk SSSR], 4:465-478, 1940. 
[23] H. Mäurer. First and second order sufficient optimality conditions in mathematical programming and optimal control. Math. Programming Stud., (14):163-177, 1981. Mathematical programming at Oberwolfach (Proc. Conf., Math. Forschungsinstitut, Oberwolfach, 1979).

[24] A. A. Milyutin and N. P. Osmolovskii. Calculus of variations and optimal control, volume 180 of Translations of Mathematical Monographs. American Mathematical Society, Providence, RI, 1998.

[25] N. P. Osmolovskii and H. Maurer. Applications to regular and bang-bang control, volume 24 of Advances in Design and Control. Society for Industrial and Applied Mathematics (SIAM), Philadelphia, PA, 2012.

[26] L. S. Pontryagin, V. G. Boltyanskii, R. V. Gamkrelidze, and E. F. Mishchenko. The mathematical theory of optimal processes. Translated from the Russian by K. N. Trirogoff; edited by L. W. Neustadt. Interscience Publishers John Wiley \& Sons, Inc. New York-London, 1962.

[27] S. M. Robinson. First order conditions for general nonlinear optimization. SIAM J. Appl. Math., 30(4):597-607, 1976.

[28] R. T. Rockafellar. Integral functionals, normal integrands and measurable selections. In Nonlinear operators and the calculus of variations (Summer School, Univ. Libre Bruxelles, Brussels, 1975), pages 157-207. Lecture Notes in Math., Vol. 543. Springer, Berlin, 1976.

[29] G. Stefani and P. Zezza. Optimality conditions for a constrained control problem. SIAM J. Control Optim., 34(2):635-659, 1996.

[30] J. Zowe and S. Kurcyusz. Regularity and stability for the mathematical programming problem in Banach spaces. Appl. Math. Optim., 5(1):49-62, 1979. 


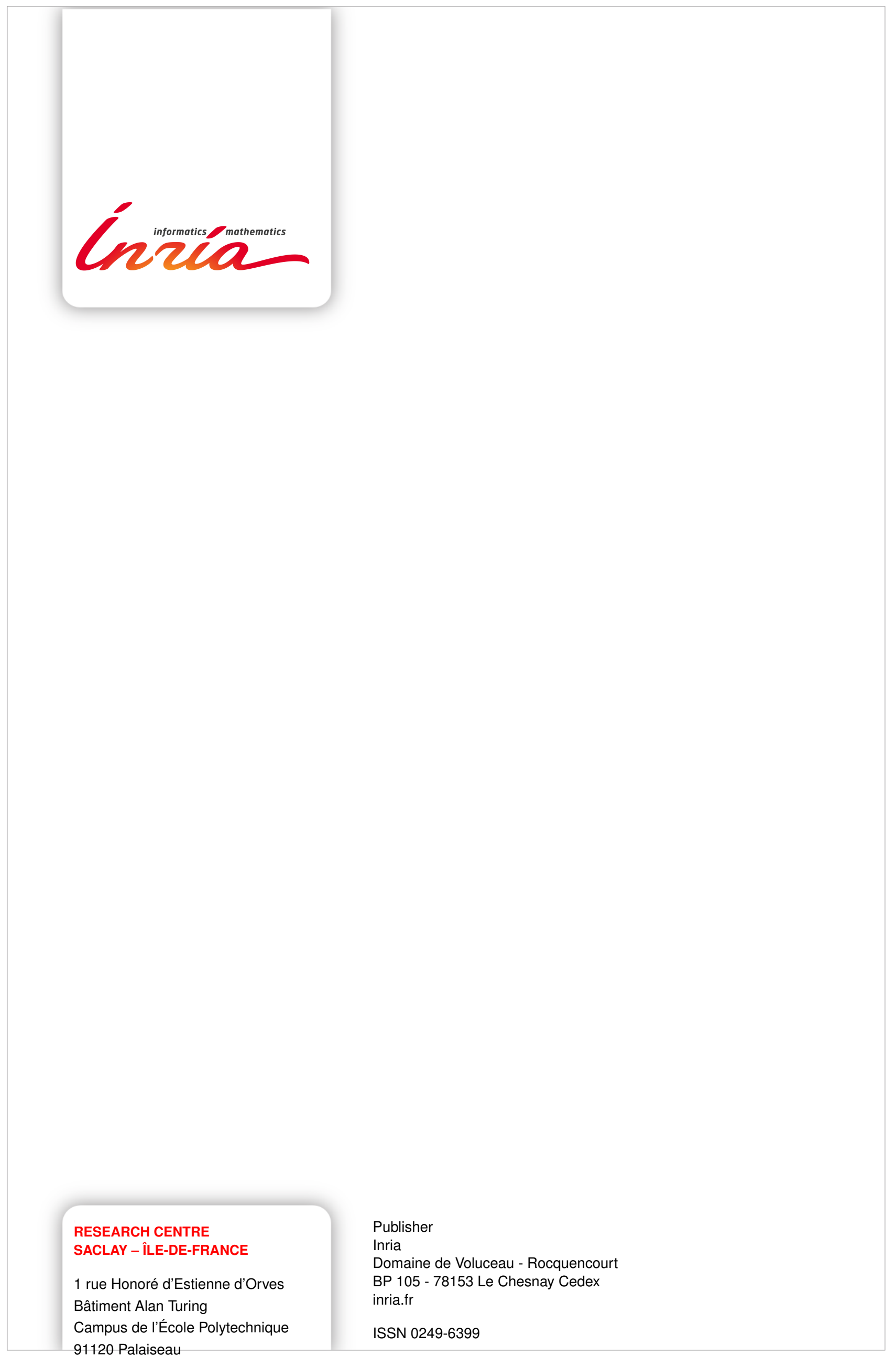

